\title{
Homogeneous Rational Lyapunov Functions for Performance Analysis of Switched Systems with Arbitrary Switching and Dwell Time Constraints
}

\author{
Graziano Chesi ${ }^{1}$, Fellow, IEEE, and Patrizio Colaneri ${ }^{2}$, Fellow, IEEE $^{2}$
}

\begin{abstract}
This paper addresses the problems of determining the $\mathcal{H}_{2}$ norm and the RMS gain of continuous-time switched linear systems. A novel class of Lyapunov functions is proposed for reaching this goal, called homogeneous rational Lyapunov functions (HRLFs). It is shown that sufficient conditions for establishing upper bounds of the sought performance indexes in the case of arbitrary switching can be given in terms of linear matrix inequality (LMI) feasibility tests by searching for an HRLF of chosen degree. Moreover, it is shown that these conditions are also necessary by searching for an HRLF of degree sufficiently large. It is worth mentioning that necessary and sufficient LMI conditions have not been proposed yet in the literature for the considered problems. Hence, the paper continues by considering the case of switching with dwell time constraints, showing that analogous LMI conditions can be obtained for this case by searching for a family of HRLFs mutually constrained by the dwell time specification. Some numerical examples illustrate the proposed methodology and highlight the advantages with respect to the existing works.
\end{abstract}

Index Terms-Switched system, $\mathcal{H}_{2}$ norm, RMS gain, LMI.

\section{INTRODUCTION}

It is well known that switched systems play a key role in automatic control. These are dynamical systems whose mathematical models are allowed to change with the time in a finite family as effect of a signal called switching policy. Switched systems naturally arise in a number of applications, and in general whenever some coefficients can vary in an admissible finite set. In this paper, the switching rule is assumed to be an exogenous deterministic signal.

Switched systems are generally classified into two main classes: switched systems with arbitrary switching and switched systems with dwell time constraints. In the former class, the changes among the mathematical models can occur arbitrarily fast. In the latter class, these changes can occur only after a minimum time, called dwell time.

A fundamental problem in automatic control consists of establishing asymptotical stability. This problem has been investigated in numerous works for switched linear systems, see for instance the books [1], [21] and the survey [23] for general results. Other works include [3], [11], [12], [17], [20], [22], [28]. The control synthesis of these systems has been studied in works such as [14], [19], [25], [27].

Another fundamental problem in automatic control consists of determining performance indexes, in particular the $\mathcal{H}_{2}$

\footnotetext{
${ }^{1}$ Graziano Chesi is with the Department of Electrical and Electronic Engineering, The University of Hong Kong. Contact information: see http://www. eee.hku.hk/ chesi

${ }^{2}$ Patrizio Colaneri is with the Dipartimento di Elettronica, Informazione e Bioingegneria (DEIB), Politecnico di Milano e IEIIT-CNR. Contact information: see http://home.dei.polimi.it/colaneri
}

norm and the root mean square (RMS) gain (also known as $\mathcal{L}_{2}$ gain or $\mathcal{H}_{\infty}$ norm), see for instance [2], [10], [29] for their definitions and calculations in the context of linear time-invariant (LTI) systems. In the context of switched linear systems, the $\mathcal{H}_{2}$ norm is defined as the maximum over all the admissible switching policies of the square root of the sum of the energies of the impulsive responses of each scalar input, and the RMS gain is defined as the maximum over all the admissible switching policies and over all the inputs of the ratio between the square roots of the energies of the output and of the input of the system for null initial conditions. These indexes have been studied for switched linear systems in [15], [16], [23], [24] through techniques such as variational principles and worst-case control. In [13], linear matrix inequality (LMI) conditions have been proposed in order to determine upper bounds through convex optimization based on the use of common and piece-wise quadratic Lyapunov functions.

Unfortunately, the existing conditions for determining performance indexes in switched linear systems through convex optimization are sufficient only. Indeed, the existing LMI conditions provide upper bounds of the sought indexes based on the use of quadratic Lyapunov functions, which are generally conservative. In this regard, it should be observed that necessary and sufficient LMI conditions have been proposed for establishing asymptotical stability of switched linear systems based on the use of non-quadratic Lyapunov functions. In particular, necessary and sufficient LMI conditions have been proposed for establishing asymptotical stability of switched linear systems based on the use of homogeneous polynomial Lyapunov functions in [5], [9] for the case of arbitrary switching, and in [8] for the case of switching with dwell time constraints.

Hence, a question arises: is it possible to exploit nonquadratic Lyapunov functions in order to derive LMI conditions that are not only sufficient but also necessary for determining performance indexes of switched linear systems? This paper aims at providing a positive answer to this question. And, interestingly, this cannot be achieved using the homogeneous polynomial Lyapunov functions that have been exploited in the literature for establishing asymptotical stability of switched linear systems, but requires the introduction of a novel class of Lyapunov functions.

Specifically, the paper addresses the problems of determining the $\mathcal{H}_{2}$ norm and the RMS gain of continuous-time switched linear systems. A novel class of Lyapunov functions is proposed for reaching this goal, called homogeneous rational Lyapunov functions (HRLFs). It is shown that sufficient conditions for establishing upper bounds of the sought performance 
indexes in the case of arbitrary switching can be given in terms of LMI feasibility tests by searching for an HRLF of chosen degree. Moreover, it is shown that these conditions are also necessary by searching for an HRLF of degree sufficiently large. Hence, the paper continues by considering the case of switching with dwell time constraints, showing that analogous LMI conditions can be obtained for this case by searching for a family of HRLFs mutually constrained by the dwell time specification. Some numerical examples illustrate the proposed methodology and highlight the advantages with respect to the existing works.

The paper is organized as follows. Section II introduces the preliminaries. Section III addresses the determination of the sought performance indexes in the case of arbitrary switching. Section IV investigates these indexes in the case of switching with dwell time constraints. Section V presents the examples. Lastly, Section VI concludes the paper with some final remarks. This paper extends our preliminary conference papers [6], [7] by including the case of switching with dwell time constraints.

\section{PRELIMINARIES}

\section{A. Notation}

$\mathbb{N}, \mathbb{R}, \mathbb{C}$ : sets of non-negative integers, real numbers, and complex numbers. $0_{n}, 0_{n \times m}: n \times 1$ and $n \times m$ null matrices. $I_{n}, I$ : identity matrices of size $n \times n$ and size specified by the context. $A^{\prime}, \operatorname{trace}(A), \operatorname{det}(A): \operatorname{transpose}$, trace, and determinant of $A$. he $(A): A+A^{\prime} \cdot \operatorname{diag}\left(A_{1}, A_{2}, \ldots\right)$ : block diagonal matrix with blocks $A_{1}, A_{2}, \ldots$ starting from the top-left corner. $A \otimes B$ : Kronecker's product between $A$ and $B$. $A^{\otimes n}: n$-th Kronecker power. $A \geq 0$ (respectively, $A>0$ ): symmetric positive semidefinite (respectively, definite) matrix A. $\|a\|_{p}: p$-norm of $a . a^{b}: a_{1}^{b_{1}} a_{2}^{b_{2}} \cdots$. $\star$ : corresponding block in a symmetric matrix. s.t.: subject to. $(a)_{i},(A)_{i, j}: i$-th entry of $a$ and $(i, j)$-th entry of $A$. Unless specified otherwise, $x_{i}$ denotes the $i$-th entry of $x$, and $X_{i, j}$ denotes the $(i, j)$-th entry of $X$. A function $v: \mathbb{R}^{n} \rightarrow \mathbb{R}$ is said to be positive semidefinite (respectively, definite) if $v\left(0_{n}\right)=0$ and $v(x) \geq 0$ (respectively, $v(x)>0$ ) for all $x \neq 0_{n}$.

\section{B. Formulation of the Problems}

Let us consider the switched system

$$
\left\{\begin{aligned}
\dot{x}(t) & =A_{\sigma(t)} x(t)+B_{\sigma(t)} w(t) \\
y(t) & =C_{\sigma(t)} x(t)+D_{\sigma(t)} w(t) \\
\sigma & \in \mathcal{D}
\end{aligned}\right.
$$

where $t \in \mathbb{R}$ is the time, $x(t) \in \mathbb{R}^{n}$ is the state, $w(t) \in \mathbb{R}^{m}$ is the input, $y(t) \in \mathbb{R}^{p}$ is the output, $\sigma: \mathbb{R} \rightarrow\{1, \ldots, N\}$ is the switching policy, and $\mathcal{D}$ is a set of switching policies of interest that will be specified in the sequel. The matrices $A_{i} \in \mathbb{R}^{n \times n}, B_{i} \in \mathbb{R}^{n \times m}, C_{i} \in \mathbb{R}^{p \times n}$ and $D_{i} \in \mathbb{R}^{p \times m}$, $i=1, \ldots, N$, are given. We say that the switched system (1) obtained for $\sigma(t)=i$ is the $i$-th subsystem of (1). It is assumed that all the subsystems of (1) are reachable and observable minimal state space realizations.
Let us denote the $\mathcal{L}_{2}$-norm of a vector signal with the notation $\|\cdot\|_{\mathcal{L}_{2}}$, i.e.,

$$
\|w\|_{\mathcal{L}_{2}}=\sqrt{\int_{0}^{\infty}\|w(t)\|_{2}^{2} d t} .
$$

The first performance index considered in this paper is the $\mathcal{H}_{2}$ norm, which is defined as follows.

Definition 1: The $\mathcal{H}_{2}$ norm of (1) is

$$
\gamma_{\mathcal{H}_{2}}=\sqrt{\sup _{\sigma(\cdot) \in \mathcal{D}} \sum_{j=1}^{m}\left\|Y_{j}\right\|_{\mathcal{L}_{2}}^{2}}
$$

where $Y_{j}(t)$ is the response of (1) due to an impulse applied to the $j$-th channel, i.e., the solution $y(t)$ in (1) for $x\left(0_{-}\right)=0_{n}$ and $w(t)$ given by

$$
w(t)=\delta(t) O_{m, j}
$$

where $\delta(t) \in \mathbb{R}$ is the Dirac function and $O_{m, j} \in \mathbb{R}^{m}$ is the $j$-th column of $I_{m}$.

The second performance index considered in this paper is the RMS gain, which is defined as follows.

Definition 2: The RMS gain of (1) is

$$
\gamma_{R M S}=\sup _{\sigma(\cdot) \in \mathcal{D}, w(\cdot)} \frac{\|y\|_{\mathcal{L}_{2}}}{\|w\|_{\mathcal{L}_{2}}}
$$

where $y(t)$ is the solution in (1) for $x\left(0^{-}\right)=0_{n}$.

The first problem addressed in this paper is as follows.

Problem 1: Determine the $\mathcal{H}_{2}$ norm and the RMS gain of (1) in the case of arbitrary switching, i.e., the indexes $\gamma_{\mathcal{H}_{2}}$ and $\gamma_{R M S}$ in (3) and (5) for $\mathcal{D}=\mathcal{D}_{a r b}$, where

$$
\mathcal{D}_{\text {arb }}=\{\sigma: \mathbb{R} \rightarrow\{1, \ldots, N\}\}
$$

is the set of switching policies with arbitrary switching.

The second problem addressed in this paper is as follows.

Problem 2: Determine the $\mathcal{H}_{2}$ norm and the RMS gain of (1) in the case of switching with dwell time constraints, i.e., the indexes $\gamma_{\mathcal{H}_{2}}$ and $\gamma_{R M S}$ in (3) and (5) for $\mathcal{D}=\mathcal{D}_{T}$, where

$$
\begin{aligned}
\mathcal{D}_{T}= & \{\sigma: \mathbb{R} \rightarrow\{1, \ldots, N\}, \sigma(t)=\text { constant } \\
& \left.\forall t \in\left[t_{i}, t_{i+1}\right), i \in \mathbb{N}, t_{i+1}-t_{i} \geq T\right\}
\end{aligned}
$$

is the set of switching policies with dwell time $T>0$.

The dependence on $t$ of the various signals will be omitted in the sequel for ease of notation unless specified otherwise. 


\section{Representation of Polynomials}

Definition 3: The function $u: \mathbb{R}^{n} \rightarrow \mathbb{R}$ is said to be a homogeneous polynomial of degree $d \in \mathbb{N}$ if

$$
u(x)=\sum_{\substack{k \in \mathbb{N}^{n} \\ k_{1}+\ldots+k_{n}=d}} a_{k} x^{k}
$$

for some $a_{k} \in \mathbb{R}$. The set of such functions is denoted by

$$
\mathcal{P}_{d}=\left\{u: \mathbb{R}^{n} \rightarrow \mathbb{R}: \text { (8) holds }\right\} .
$$

\section{A. HRLFs}

Definition 4: The function $v: \mathbb{R}^{n} \rightarrow \mathbb{R}$ is said to be a homogeneous rational function of total degree $r \in \mathbb{N}$ and relative degree $s \in \mathbb{N}$, with $r \geq s$, if

$$
\left\{\begin{aligned}
v(x) & =\frac{\phi(x)}{\psi(x)} \\
\phi & \in \mathcal{P}_{r} \\
\psi & \in \mathcal{P}_{r-s} .
\end{aligned}\right.
$$

The set of such functions is denoted by

$$
\mathcal{Q}_{r, s}=\left\{v: \mathbb{R}^{n} \rightarrow \mathbb{R},(15) \text { holds }\right\} .
$$

Let us denote with $b(x, d)$ a vector base for $\mathcal{P}_{d}$. For instance, $b(x, d)$ can be chosen as a vector whose entries are all the monomials in $x \in \mathbb{R}^{n}$ of degree $d \in \mathbb{N}$ with unitary coefficient without repetitions. Such a vector can be obtained according to the recursive rule

$$
b(x, d)=\left\{\begin{array}{cc}
1 & \text { if } d=0 \\
\left(\begin{array}{c}
x_{1} b\left(X_{1}, d-1\right) \\
\vdots \\
x_{n} b\left(X_{n}, d-1\right)
\end{array}\right) & \text { if } d>0
\end{array}\right.
$$

where

$$
X_{i}=\left(\begin{array}{c}
x_{i} \\
\vdots \\
x_{n}
\end{array}\right)
$$

The length of $b(x, d)$ is given by

$$
c(n, d)=\frac{(n+d-1) !}{(n-1) ! d !} .
$$

For reasons that will become clear in the sequel, we also define $b(x, d)$ for negative values of $d$ as follows:

$$
b(x, d)=0 \quad \forall d<0 .
$$

By using $b(x, d)$, a homogeneous polynomial $v(x)$ of degree $2 d$ can be expressed as

$$
v(x)=b(x, d)^{\prime} V b(x, d)
$$

for some $V \in \mathbb{R}^{c(n, d) \times c(n, d)}, V=V^{\prime}$. The representation (14) is known as Gram matrix method or square matricial representation (SMR), see for instance the survey [4].

\section{ARbitrary SWITCHING}

This section addresses Problem 1, i.e., the determination of the sought performance indexes in the case of arbitrary switching. In particular, Section III-A introduces the novel class of Lyapunov functions, Section III-B presents the conditions for establishing upper bounds on the $\mathcal{H}_{2}$ norm, and Section III-C presents the conditions for establishing upper bounds on the RMS gain.
The novel class of Lyapunov functions introduced in this paper is defined as follows.

Definition 5: The function $v: \mathbb{R}^{n} \rightarrow \mathbb{R}$ is said to be a homogeneous rational Lyapunov function (HRLF) for (1) (with null input $w$ ) if:

1) $v \in \mathcal{Q}_{r, s}$ where $r$ and $s$ are even and such that $r \geq s \geq$ 2

2) $v(x)$ can be expressed as in (15) with $\phi(x)$ positive definite and $\psi(x)$ positive for all $x \neq 0_{n}$;

$$
\dot{v}(x)=\nabla v(x) A_{\sigma} x .
$$

In particular, such a function $v(x)$ is said to be an HRLF of total degree $r$ and relative degree $s$.

It is useful to observe that, if $v(x)$ is an HRLF, then:

1) the numerator $\phi(x)$ is a positive definite homogeneous polynomial of even degree $r$, where $r \geq 2$. This also implies that $\phi(x)$ cannot be a constant;

2) the denominator $\psi(x)$ is a homogeneous polynomial of even degree $r-s$, where $0 \leq r-s \leq r-2$. In particular, $\psi(x)$ is a:

a) positive constant if $r=s$;

b) positive definite homogeneous polynomial if $r>s$;

3) $v(x)$ is homogeneous of degree $s$, i.e.,

$$
v(\alpha x)=\alpha^{s} v(x) \quad \forall \alpha \in \mathbb{R}
$$

since the numerator and denominator of $v(x)$ are homogeneous polynomials of degree $r$ and $r-s$;

4) $v(x)$ is radially unbounded, continuous and positive definite. Indeed, $v(x)$ is radially unbounded since the relative degree $s$ is positive. Moreover, $v(x)$ is continuous and positive definite because it is the ratio of polynomials that are positive for all $x \neq 0_{n}$ and because

$$
\lim _{x \rightarrow 0_{n}} v(x)=\lim _{\alpha \rightarrow 0} \alpha^{s} v(\tilde{x})=0
$$

where $\tilde{x} \neq 0_{n}$ is arbitrary;

5) $v(x)$ is continuously differentiable. Indeed, one has

$$
\nabla v(x)=\frac{\psi(x) \nabla \phi(x)-\phi(x) \nabla \psi(x)}{\psi(x)^{2}}
$$

3) $\dot{v}(x)$ is negative definite for all $\sigma$, where 
which is continuous because $\phi(x)$ and $\psi(x)$ are continuous, $\psi(x)$ is positive for all $x \neq 0_{n}$, and

$$
\lim _{x \rightarrow 0_{n}} \nabla v(x)=\lim _{\alpha \rightarrow 0} \alpha^{s-1} \nabla v(\tilde{x})=0_{n}
$$

where $\tilde{x} \neq 0_{n}$ is arbitrary.

Throughout the paper we will aim at searching for HRLFs of total degree $2 d, d \in \mathbb{N}$ with $d \geq 1$, and relative degree 2 , i.e., in the set $\mathcal{Q}_{2 d, 2}$.

Let us observe that any HRLF $v(x)$ in the set $\mathcal{Q}_{2 d, 2}$ can be bounded from above and below by quadratic functions over compact sets, i.e., for all compact sets $\mathcal{S} \subset \mathbb{R}^{n}$ there exist $Q_{1}>0$ and $Q_{2}>0$ such that

$$
x^{\prime} Q_{1} x \leq v(x) \leq x^{\prime} Q_{2} x \quad \forall x \in \mathbb{R}^{n} .
$$

For instance, one can select

$$
\begin{cases}Q_{1}=\rho_{1} I_{n}, & \rho_{1}=\min _{x \in \mathcal{S}} \frac{v(x)}{\|x\|_{2}^{2}} \\ Q_{2}=\rho_{2} I_{n}, & \rho_{2}=\max _{x \in \mathcal{S}} \frac{v(x)}{\|x\|_{2}^{2}}\end{cases}
$$

where $\rho_{1}$ and $\rho_{2}$ are positive and finite since $\mathcal{S}$ is compact and $\frac{v(x)}{\|x\|_{2}^{2}}$ is a homogeneous rational function where the numerator and denominator are positive definite homogeneous polynomials of the same degree.

Also, let us observe that HRLFs in the set $\mathcal{Q}_{2 d, 2}$ contain quadratic Lyapunov functions as a special case. Indeed, the numerator and denominator of $v \in \mathcal{Q}_{2 d, 2}$ are a homogeneous polynomial of degree $2 d$ and a homogeneous polynomial of degree $2 d-2$. Hence, for $d=1$, HRLFs are quadratic Lyapunov functions since the numerator and denominator of $v \in \mathcal{Q}_{2,2}$ are a quadratic function and a constant.

Let $v(x)$ be an HRLF candidate in the set $\mathcal{Q}_{2 d, 2}$. By exploiting the representation introduced in Section II-C, $v(x)$ can be expressed as

$$
\left\{\begin{aligned}
v(x) & =\frac{\phi(x)}{\psi(x)} \\
\phi(x) & =b(x, d)^{\prime} \Phi b(x, d) \\
\psi(x) & =b(x, d-1)^{\prime} \Psi b(x, d-1)
\end{aligned}\right.
$$

where $\Phi \in \mathbb{R}^{c(n, d) \times c(n, d)}, \quad \Phi=\Phi^{\prime}, \quad$ and $\Psi \in \mathbb{R}^{c(n, d-1) \times c(n, d-1)}, \Psi=\Psi^{\prime}$.

Hereafter we describe the proposed methodology in the case of arbitrary switching. In particular, a Lyapunov function common to all the subsystems of (1) is searched for. In this respect, it is useful to observe that common Lyapunov functions are guaranteed to exist for asymptotically stable switched systems in the case of arbitrary switching, see for instance [1].

\section{B. Arbitrary Switching: $\mathcal{H}_{2}$ Norm}

In this section we show how HRLFs can be used to determine the $\mathcal{H}_{2}$ norm of (1) in the case of arbitrary switching. Let us observe that a necessary condition for the $\mathcal{H}_{2}$ norm to be finite is that (1) is strictly proper, i.e.,

$$
D_{i}=0 \quad \forall i=1, \ldots, N .
$$

For $s=0,1$, let $A_{i, s}$ be the matrix satisfying

$$
\frac{d b(x, d-s)}{d x} A_{i} x=A_{i, s} b(x, d-s) .
$$

For $\Phi$ and $\Psi$ as in (24), let us define

$$
\left\{\begin{aligned}
E_{i, 1}(\Phi) & =J_{1}^{\prime}\left(\text { he }\left(\Phi A_{i, 0}\right) \otimes \Psi\right) J_{1} \\
E_{i, 2}(\Phi) & =J_{1}^{\prime}\left(\Phi \otimes \text { he }\left(\Psi A_{i, 1}\right)\right) J_{1} \\
E_{i, 3} & =J_{2}^{\prime}\left(\Psi^{\otimes 2} \otimes C_{i}^{\prime} C_{i}\right) J_{2}
\end{aligned}\right.
$$

where $J_{1}$ and $J_{2}$ are the matrices satisfying

$$
\left\{\begin{aligned}
b(x, d) \otimes b(x, d-1) & =J_{1} b(x, 2 d-1) \\
b(x, d-1)^{\otimes 2} \otimes x & =J_{2} b(x, 2 d-1) .
\end{aligned}\right.
$$

Let us define

$$
E_{i}(\Phi)=E_{i, 1}(\Phi)-E_{i, 2}(\Phi)+E_{i, 3} .
$$

Let us express $B_{i}$ as

$$
B_{i}=\left(B_{i, 1}, \ldots, B_{i, m}\right)
$$

where $B_{i, 1}, \ldots, B_{i, m} \in \mathbb{R}^{n}$, and let us define

$$
g_{i}(\Phi)=\sum_{j=1}^{m} \frac{b\left(B_{i, j}, d\right)^{\prime} \Phi b\left(B_{i, j}, d\right)}{b\left(B_{i, j}, d-1\right)^{\prime} \Psi b\left(B_{i, j}, d-1\right)} .
$$

Lastly, let $M_{1}(\Theta)$ be a linear parametrization of the set

$$
\mathcal{M}_{1}=\left\{M=M^{\prime}: b(x, 2 d-1)^{\prime} M b(x, 2 d-1)=0\right\}
$$

where $\Theta$ is a free vector. The following result provides an LMI condition for establishing upper bounds of $\gamma_{\mathcal{H}_{2}}$ in the case $\mathcal{D}=\mathcal{D}_{\text {arb }}$.

Theorem 1: Consider the case of arbitrary switching, i.e., $\mathcal{D}=\mathcal{D}_{\text {arb }}$. Let $d \geq 1$ and $\Psi>0$ be chosen. Suppose that there exist $\xi, \Phi$ and $\Theta_{i}, i=1, \ldots, N$, satisfying the system of LMIs

$$
\left\{\begin{aligned}
0 & <\Phi \\
0 & >E_{i}(\Phi)+M_{1}\left(\Theta_{i}\right) \quad \forall i=1, \ldots, N \\
0 & <\xi-g_{i}(\Phi) \quad \forall i=1, \ldots, N .
\end{aligned}\right.
$$

Then, $v(x)$ in (24) is an HRLF for (1), and one has

$$
\gamma_{\mathcal{H}_{2}}<\sqrt{\xi} .
$$

Proof. Suppose that the system of LMIs (33) holds. From the definition of $b(x, d)$ it follows that

$$
b(x, d) \neq 0 \quad \forall x \neq 0_{n} .
$$


Hence, from (24), the first inequality in (33) and the fact that $\Psi>0$, one has

$$
\forall x \neq 0_{n}\left\{\begin{array}{l}
\phi(x)>0 \\
\psi(x)>0
\end{array}\right.
$$

since $d$ is arbitrary. By pre- and post-multiplying the second inequality in (33) times $b(x, 2 d-1)^{\prime}$ and $b(x, 2 d-1)$, it follows that

$$
\begin{gathered}
0>b(x, 2 d-1)^{\prime}\left(E_{i}(\Phi)+M_{1}\left(\Theta_{i}\right)\right) b(x, 2 d-1) \\
\forall x \neq 0_{n} .
\end{gathered}
$$

Since $M_{1}(\theta)$ is a linear parametrization of the set $\mathcal{M}_{1}$ in (32), one has

$$
b(x, 2 d-1)^{\prime} M_{1}\left(\Theta_{i}\right) b(x, 2 d-1)=0
$$

and, hence,

$$
\begin{gathered}
0>b(x, 2 d-1)^{\prime}\left(E_{i, 1}(\Phi)-E_{i, 2}(\Phi)+E_{i, 3}\right) b(x, 2 d-1) \\
\forall x \neq 0_{n} .
\end{gathered}
$$

Let us observe that

$$
\begin{aligned}
& b(x, 2 d-1)^{\prime} E_{i, 1}(\Phi) b(x, 2 d-1) \\
= & b(x, 2 d-1)^{\prime} J_{1}^{\prime}\left(\text { he }\left(\Phi A_{i, 0}\right) \otimes \Psi\right) J_{1} b(x, 2 d-1) \\
= & (b(x, d) \otimes b(x, d-1))^{\prime}\left(\text { he }\left(\Phi A_{i, 0}\right) \otimes \Psi\right) \\
& \cdot(b(x, d) \otimes b(x, d-1)) \\
= & \left(b(x, d)^{\prime} \operatorname{he}\left(\Phi A_{i, 0}\right) b(x, d)\right) \\
& \otimes\left(b(x, d-1)^{\prime} \Psi b(x, d-1)\right) \\
= & \psi(x) \nabla \phi(x) A_{i} x .
\end{aligned}
$$

Proceeding with similar calculations, one gets

$$
\begin{gathered}
0>\psi(x) \nabla \phi(x) A_{i} x-\phi(x) \nabla \psi(x) A_{i} x+\psi(x)^{2}\left\|C_{i} x\right\|_{2}^{2} \\
\forall x \neq 0_{n}
\end{gathered}
$$

which, dividing by $\psi(x)^{2}$, implies

$$
0>\nabla v(x) A_{i} x+\left\|C_{i} x\right\|_{2}^{2} \quad \forall x \neq 0_{n} .
$$

Hence, $v(x)$ is an HRLF. By replacing $i$ with $\sigma$ in the last inequality, and integrating both sides of this inequality with respect to $t$ from 0 to $\infty$, one gets

$$
0>v(x(\infty))-v(x(0))+\int_{0}^{\infty}\left\|C_{\sigma} x\right\|_{2}^{2} d t
$$

that, given the nonnegativity of $v(x(\infty))$, implies

$$
v(x(0))>\left\|C_{\sigma} x\right\|_{\mathcal{L}_{2}}^{2} .
$$

At this point, let us suppose that the input $w$ is chosen as in (4). It follows that

$$
x(0)=B_{\sigma(0)} O_{m, j} .
$$

Moreover, in such a case, the response of (1) is

$$
y=C_{\sigma} x=Y_{j}
$$

where $Y_{j}$ is the impulse response in Definition 1. Hence,

$$
v\left(B_{\sigma(0)} O_{m, j}\right)>\left\|Y_{j}\right\|_{\mathcal{L}_{2}}^{2} .
$$

Summing up over $j=1, \ldots, m$ and taking into account that $\sigma(0)$ is arbitrary, the result follows.

The LMI condition provided by Theorem 1 allows one to establish upper bounds of the $\mathcal{H}_{2}$ norm of (1) in the case of arbitrary switching. This condition is sufficient for any a priori chosen positive integer $d$ (which defines the total degree of the HRLF, equal to $2 d$ ) and positive definite matrix $\Psi$ (which defines the denominator of the HRLF, according to (24)).

The matrices used to define the LMI condition (33) can be easily built, see the Appendix for details. Let us observe that the set $\mathcal{M}_{1}$ may contain only the null matrix (this happens when $d=1$ ). In such a case, the linear parametrization $M_{1}(\cdot)$ is the null matrix itself, and the vectors $\Theta_{i}$ in Theorem 1 are empty vectors (i.e., do not need to be introduced).

The best upper bound provided by Theorem 1 for chosen $d$ and $\Psi$ can be found by minimizing $\xi$ subject to the LMIs (33), and is equal to

$$
\hat{\gamma}_{\mathcal{H}_{2}}=\sqrt{\xi_{\mathcal{H}_{2}}}
$$

where $\xi_{\mathcal{H}_{2}}$ is the solution of the semidefinite program (SDP)

$$
\begin{aligned}
\xi_{\mathcal{H}_{2}}= & \inf _{\xi, \Phi, \Theta_{i}} \xi \\
& \text { s.t. (33) holds. }
\end{aligned}
$$

Indeed, from Theorem 1 it follows that

$$
\gamma_{\mathcal{H}_{2}} \leq \hat{\gamma}_{\mathcal{H}_{2}} .
$$

The following algorithm summarizes the computation of the upper bound $\hat{\gamma}_{\mathcal{H}_{2}}$ of the $\mathcal{H}_{2}$ norm of (1):

1) choose an integer $d \geq 1$ and a matrix $\Psi>0$;

2) compute the matrices $A_{i, 0}, A_{i, 1}, J_{1}$ and $J_{2}$;

$3)$ compute the matrix functions $E_{i}(\Phi), M_{1}\left(\Theta_{i}\right)$ and $g_{i}(\Phi)$

4) solve the $\operatorname{SDP}(36)$;

5) get the upper bound $\hat{\gamma}_{\mathcal{H}_{2}}$ from (35).

The following result states that the LMI condition provided by Theorem 1 is not only sufficient but also necessary by using sufficiently large values of $d$.

Theorem 2: Consider the case of arbitrary switching, i.e., $\mathcal{D}=\mathcal{D}_{\text {arb }}$. Suppose without loss of generality that $\gamma_{\mathcal{H}_{2}}<\infty$. Then, for all $\varepsilon>0$ there exists $d$ such that (33) holds for some $\xi, \Phi$ and $\Theta_{i}$, with $\Psi$ satisfying

$$
\left\{\begin{aligned}
\Psi & >0 \\
\psi(x) & =\|x\|_{2}^{2 d-2}
\end{aligned}\right.
$$

and

$$
\gamma_{\mathcal{H}_{2}}<\sqrt{\xi} \leq \gamma_{\mathcal{H}_{2}}+\varepsilon
$$

Proof. Let us start by observing that one can suppose without loss of generality that $\gamma_{\mathcal{H}_{2}}<\infty$ because, if $\gamma_{\mathcal{H}_{2}}=\infty$, then the only possible upper bound of $\gamma_{\mathcal{H}_{2}}$ provided by Theorem 1 in (34) is $\sqrt{\xi}=\infty$, i.e., the upper bound coincides with the sought $\mathcal{H}_{2}$ norm. 
Hence, let us suppose $\gamma_{\mathcal{H}_{2}}<\infty$. Let $\varepsilon>0$ and $\xi$ be such that (39) holds. Let $z: \mathbb{R}^{n} \rightarrow \mathbb{R}$ be any Lyapunov function proving that the $\mathcal{H}_{2}$ norm of (1) is smaller than $\sqrt{\xi}$, i.e.,

$$
\forall i=1, \ldots, N\left\{\begin{aligned}
0 & <z(x) \\
0 & >\nabla z(x) A_{i} x+\left\|C_{i} x\right\|_{2}^{2} \\
0 & <\xi-\sum_{j=1}^{m} z\left(B_{i, j}\right) .
\end{aligned}\right.
$$

This Lyapunov function can be constructed based on the worst solution of the $\mathcal{H}_{2}$ differential Lyapunov equation, see for details [13], [18], [24] adapted to the $\mathcal{H}_{2}$ case. Since (1) is linear, without loss of generality one can suppose that $z(x)$ is homogeneous of degree 2, i.e.,

$$
z(\alpha x)=\alpha^{2} z(x) \quad \forall \alpha \in \mathbb{R} .
$$

Let us define the hyper-sphere of dimension $n$ with center in the origin and radius one as

$$
\mathcal{B}=\left\{x \in \mathbb{R}^{n}:\|x\|_{2}=1\right\} .
$$

Since $\mathcal{B}$ is compact and $z(x)$ is continuous over $\mathcal{B}, z(x)$ can be approximated arbitrarily well over $\mathcal{B}$ by polynomial functions. This means that, for all $\varepsilon_{1}>0$, there exists a polynomial $z_{1}(x)$ such that

$$
\left|z(x)-z_{1}(x)\right| \leq \varepsilon_{1} \quad \forall x \in \mathcal{B} .
$$

Without loss of generality, one can assume that $z_{1}(x)$ is homogeneous of degree $2 d_{1}$ with $d_{1} \in \mathbb{N}, d_{1} \geq 1$. Since $\|x\|_{2}=1$ over $\mathcal{B}$, the previous condition can be rewritten as

$$
\left|z(x)-\frac{z_{1}(x)}{z_{2}(x)}\right| \leq \varepsilon_{1} \quad \forall x \in \mathcal{B}
$$

where

$$
z_{2}(x)=\|x\|_{2}^{2\left(d_{1}-1\right)} .
$$

Since $\varepsilon_{1}$ is arbitrary, one can choose it sufficiently small to ensure that

$$
\forall i=1, \ldots, N\left\{\begin{aligned}
0 & <z_{3}(x) \\
0 & >\nabla z_{3}(x) A_{i} x+\left\|C_{i} x\right\|_{2}^{2} \\
0 & <\xi-\sum_{j=1}^{m} z_{3}\left(B_{i, j}\right)
\end{aligned}\right.
$$

where

$$
z_{3}(x)=\frac{z_{1}(x) z_{4}(x)}{z_{2}(x) z_{4}(x)}
$$

and $z_{4}(x)$ is a function that can be arbitrarily chosen. This means that

$$
\forall i=1, \ldots, N\left\{\begin{aligned}
0< & z_{1}(x) z_{4}(x) \\
0> & z_{4}(x)^{2}\left(\left(z_{2}(x) \nabla z_{1}(x)\right.\right. \\
& \left.-z_{1}(x) \nabla z_{2}(x)\right) A_{i} x \\
& \left.+z_{2}(x)^{2}\left\|C_{i}^{\prime} x\right\|_{2}^{2}\right) \\
0< & \xi-\sum_{j=1}^{m} \frac{z_{1}\left(B_{i, j}\right) z_{4}\left(B_{i, j}\right)}{z_{2}\left(B_{i, j}\right) z_{4}\left(B_{i, j}\right)} .
\end{aligned}\right.
$$

Since $z_{4}(x)$ is arbitrary, it can be chosen of the form $\|x\|_{2}^{2} \tilde{d}$ with $\tilde{d} \in \mathbb{N}$ in order to ensure that the right hand sides of the inequalities in the previous condition, with the second one changed in sign, admit positive definite Gram matrices, see for instance [4]. This implies that there exists $\Phi>0$ satisfying (24) for

$$
\phi(x)=z_{1}(x)\|x\|_{2}^{2 \tilde{d}}
$$

which is a homogeneous polynomial of degree $2 d$ with

$$
d=d_{1}+\tilde{d} .
$$

Moreover, there exists $\Psi$ satisfying (38) since such a matrix can be simply chosen diagonal with positive entries on the diagonal. At this point, the proof proceeds by reverting the proof of Theorem 1 and observing that the existence of $\Theta_{1}, \ldots, \Theta_{N}$ satisfying (33) is ensured by $z_{4}(x)$.

Theorem 2 states that the LMI condition provided by Theorem 1 can be used to approximate arbitrarily well the $\mathcal{H}_{2}$ norm of (1) in the case of arbitrary switching by increasing $d$. This means that, for all $\varepsilon>0$, there exists $d$ such that the upper bound $\hat{\gamma}_{\mathcal{H}_{2}}$ in (35) satisfies

$$
\gamma_{\mathcal{H}_{2}} \leq \hat{\gamma}_{\mathcal{H}_{2}} \leq \gamma_{\mathcal{H}_{2}}+\varepsilon
$$

at least when $\Psi$ is chosen according to (38).

\section{Arbitrary Switching: RMS Gain}

In this section we show how HRLFs can be used to determine the RMS gain of (1) in the case of arbitrary switching. For $d \in \mathbb{N}$ let us define

$$
q(x, d, w)=b(x, d-1) \otimes w .
$$

For $s=0,1$, let $B_{i, s}$ be the matrix satisfying

$$
\frac{d b(x, d-s)}{d x} B_{i} w=B_{i, s} q(x, d-s, w) .
$$

Let $A_{i, s}$ be as in (26) and, for $\Phi$ and $\Psi$ as in (24), let us define

$$
\left\{\begin{aligned}
F_{i, 1}(\Phi) & =J_{1}^{\prime}\left(\Phi B_{i, 0} \otimes \Psi\right) J_{3} \\
F_{i, 2}(\Phi) & =J_{1}^{\prime}\left(\Phi \otimes \Psi B_{i, 1}\right) J_{4} \\
F_{i, 3} & =J_{5}^{\prime}\left(\Psi^{\otimes 2} \otimes I_{m}\right) J_{5} \\
G_{i, 1} & =J_{2}^{\prime}\left(\Psi^{\otimes 2} \otimes C_{i}^{\prime} C_{i}\right) J_{2} \\
G_{i, 2} & =J_{2}^{\prime}\left(\Psi^{\otimes 2} \otimes C_{i}^{\prime} D_{i}\right) J_{5} \\
G_{i, 3} & =J_{5}^{\prime}\left(\Psi^{\otimes 2} \otimes D_{i}^{\prime} D_{i}\right) J_{5}
\end{aligned}\right.
$$

where $J_{1}, J_{2}$ are given by (28), and $J_{3}, J_{4}, J_{5}$ are the matrices satisfying

$$
\left\{\begin{aligned}
b(x, d-1) \otimes w \otimes b(x, d-1) & =J_{3} q(x, 2 d-1, w) \\
b(x, d) \otimes b(x, d-2) \otimes w & =J_{4} q(x, 2 d-1, w) \\
b(x, d-1)^{\otimes 2} \otimes w & =J_{5} q(x, 2 d-1, w) .
\end{aligned}\right.
$$

For $\xi \in \mathbb{R}$, let us define

$$
F_{i}(\Phi, \xi)=\left(\begin{array}{cc}
E_{i, 1}(\Phi)-E_{i, 2}(\Phi) & F_{i, 1}(\Phi)-F_{i, 2}(\Phi) \\
\star & -\xi F_{i, 3}
\end{array}\right)
$$


and

$$
G_{i}=\left(\begin{array}{cc}
G_{i, 1} & G_{i, 2} \\
\star & G_{i, 3}
\end{array}\right) .
$$

Lastly, let us define

$$
r(x, d, w)=\left(\begin{array}{c}
b(x, 2 d-1) \\
b(x, 2 d-2) \otimes w
\end{array}\right)
$$

and let $M_{2}(\Theta)$ be a linear parametrization of the set

$$
\mathcal{M}_{2}=\left\{M=M^{\prime}: r(x, d, w)^{\prime} M r(x, d, w)=0\right\}
$$

where $\Theta$ is a free vector. The following result provides an LMI condition for establishing upper bounds of $\gamma_{R M S}$ in the case $\mathcal{D}=\mathcal{D}_{\text {arb }}$.

Theorem 3: Consider the case of arbitrary switching, i.e., $\mathcal{D}=\mathcal{D}_{\text {arb }}$. Let $d \geq 1$ and $\Psi>0$ be chosen. Suppose that there exist $\xi, \Phi$ and $\Theta_{i}, i=1, \ldots, N$, satisfying the system of LMIs

$$
\left\{\begin{array}{l}
0<\Phi \\
0>F_{i}(\Phi, \xi)+G_{i}+M_{2}\left(\Theta_{i}\right) \quad \forall i=1, \ldots, N .
\end{array}\right.
$$

Then, $v(x)$ in (24) is an HRLF for (1) and

$$
\gamma_{R M S}<\sqrt{\xi}
$$

Proof. Suppose that the system of LMIs (49) holds. Analogously to the proof of Theorem 1, one has

$$
\forall x \neq 0_{n}\left\{\begin{aligned}
b(x, d) & \neq 0 \\
\phi(x) & >0 \\
\psi(x) & >0
\end{aligned}\right.
$$

Similarly, from (47) it follows that

$$
r(x, d, w) \neq 0 \quad \forall x \neq 0_{n} .
$$

Hence, by pre- and post-multiplying the second inequality in (49) times $r(x, d, w)^{\prime}$ and $r(x, d, w)$, it follows that

$$
\begin{gathered}
0>r(x, d, w)^{\prime}\left(F_{i}(\Phi, \xi)+G_{i}+M_{2}\left(\Theta_{i}\right)\right) r(x, d, w) \\
\forall x \neq 0_{n} .
\end{gathered}
$$

Since $M_{2}(\theta)$ is a linear parametrization of the set $\mathcal{M}_{2}$ in (48), it follows that

$$
r(x, d, w)^{\prime} M_{2}\left(\Theta_{i}\right) r(x, d, w)=0
$$

and, hence,

$$
\begin{aligned}
& 0>b(x, 2 d-1)^{\prime}\left(E_{i, 1}(\Phi)-E_{i, 2}(\Phi)+G_{i, 1}\right) b(x, 2 d-1) \\
& +2 b(x, 2 d-1)^{\prime}\left(F_{i, 1}(\Phi)-F_{i, 2}(\Phi)+G_{i, 2}\right) q(x, 2 d-1, w) \\
& +q(x, 2 d-1, w)^{\prime}\left(G_{i, 3}-\xi F_{i, 3}\right) q(x, 2 d-1, w) \quad \forall x \neq 0_{n} .
\end{aligned}
$$

Analogously to the proof of Theorem 1, one has

$$
b(x, 2 d-1)^{\prime} E_{i, 1}(\Phi) b(x, 2 d-1)=\nabla \phi(x) A_{i} x \psi(x) .
$$

Proceeding with similar calculations, one gets

$0>\nabla \phi(x)\left(A_{i} x+B_{i} w\right) \psi(x)-\nabla \psi(x)\left(A_{i} x+B_{i} w\right) \phi(x)$ $+\left\|C_{i} x+D_{i} w\right\|_{2}^{2} \psi(x)^{2}-\xi\|w\|_{2}^{2} \quad \forall x \neq 0_{n}$ which, dividing by $\psi(x)^{2}$, implies

$$
\begin{aligned}
& 0>\nabla v(x)\left(A_{i} x+B_{i} w\right)+\left\|C_{i} x+D_{i} w\right\|_{2}^{2}-\xi\|w\|_{2}^{2} \\
& \forall x \neq 0_{n} .
\end{aligned}
$$

Hence, $v(x)$ is an HRLF. By replacing $i$ with $\sigma$ in the last inequality, and integrating both sides of this inequality with respect to $t$ from 0 to $\infty$ with the initial condition $x(0)=0_{n}$, one gets

$$
0>v(x(\infty))+\int_{0}^{\infty}\left(\|y\|_{2}^{2}-\xi\|w\|_{2}^{2}\right) d t .
$$

Since $v(x(\infty))$ is non-negative, it follows that (50) holds.

Theorem 3 provides a sufficient LMI condition for establishing upper bounds of the RMS gain of (1) in the case of arbitrary switching. The matrices used to define the LMI condition (49) can be easily built, see the Appendix for details.

The best upper bound provided by Theorem 3 for chosen $d$ and $\Psi$ can be found by minimizing $\xi$ subject to the LMIs (49), and is equal to

$$
\hat{\gamma}_{R M S}=\sqrt{\xi_{R M S}}
$$

where $\xi_{R M S}$ is the solution of the SDP

$$
\begin{aligned}
\xi_{R M S}= & \inf _{\xi, \Phi, \Theta_{i}} \xi \\
& \text { s.t. (49) holds. }
\end{aligned}
$$

Indeed, from Theorem 3 it follows that

$$
\gamma_{R M S} \leq \hat{\gamma}_{R M S}
$$

The following result states that the LMI condition provided by Theorem 3 is not only sufficient but also necessary by using sufficiently large values of $d$.

Theorem 4: Consider the case of arbitrary switching, i.e., $\mathcal{D}=\mathcal{D}_{\text {arb }}$. Suppose without loss of generality that $\gamma_{R M S}<$ $\infty$. Then, for all $\varepsilon>0$ there exists $d$ such that (49) holds for some $\xi, \Phi$ and $\Theta_{i}$, with $\Psi$ satisfying (38), and

$$
\gamma_{R M S}<\sqrt{\xi} \leq \gamma_{R M S}+\varepsilon
$$

Proof. Let us start by observing that one can suppose without loss of generality that $\gamma_{R M S}<\infty$ because, if $\gamma_{R M S}=\infty$, then the only possible upper bound of $\gamma_{R M S}$ provided by Theorem 3 in (50) is $\sqrt{\xi}=\infty$, i.e., the upper bound coincides with the sought RMS gain.

Hence, let us suppose $\gamma_{R M S}<\infty$. Let $\varepsilon>0$ and $\xi$ be such that (54) holds. Let $z: \mathbb{R}^{n} \rightarrow \mathbb{R}$ be any Lyapunov function proving that the RMS gain of (1) is smaller than $\sqrt{\xi}$, i.e.,

$$
\forall x \neq 0_{n}\left\{\begin{aligned}
0< & z(x) \\
0> & \nabla z(x)\left(A_{i} x+B_{i} w\right)+\left\|C_{i} x+D_{i} w\right\|_{2}^{2} \\
& -\xi\|w\|_{2}^{2} \forall i=1, \ldots, N .
\end{aligned}\right.
$$

This Lyapunov function can be constructed based on the solution of the $\mathcal{H}_{\infty}$ differential Riccati equation, see [13], [18], [24] for details. Analogously to the proof of Theorem $2, z(x)$ can be assumed homogeneous of degree 2 without 
loss of generality. Moreover, for all $\varepsilon_{1}>0$, there exists a homogeneous polynomial $z_{1}(x)$ of degree $2 d_{1}$ with $d_{1} \in \mathbb{N}$, $d_{1} \geq 1$, such that

$$
\left|z(x)-\frac{z_{1}(x)}{z_{2}(x)}\right| \leq \varepsilon_{1} \quad \forall x \in \mathcal{B}
$$

where $\mathcal{B}$ the hyper-sphere of dimension $n$ with center in the origin and radius one, and

$$
z_{2}(x)=\|x\|_{2}^{2\left(d_{1}-1\right)} .
$$

Since $\varepsilon_{1}$ is arbitrary, one can choose it sufficiently small to ensure that

$$
\forall x \neq 0_{n}\left\{\begin{aligned}
0< & z_{3}(x) \\
0> & \nabla z_{3}(x)\left(A_{i} x+B_{i} w\right)+\left\|C_{i} x+D_{i} w\right\|_{2}^{2} \\
& -\xi\|w\|_{2}^{2} \quad \forall i=1, \ldots, N
\end{aligned}\right.
$$

where

$$
z_{3}(x)=\frac{z_{1}(x) z_{4}(x)}{z_{2}(x) z_{4}(x)}
$$

and $z_{4}(x)$ is a function that can be arbitrarily chosen. This means that

$$
\forall x \neq 0_{n}\left\{\begin{aligned}
0< & z_{1}(x) z_{4}(x) \\
0> & z_{4}(x)^{2}\left(\left(z_{2}(x) \nabla z_{1}(x)-z_{1}(x) \nabla z_{2}(x)\right)\right. \\
& \cdot\left(A_{i} x+B_{i} w\right)+z_{2}(x)^{2}\left(\left\|C_{i} x+D_{i} w\right\|_{2}^{2}\right. \\
& \left.\left.-\xi\|w\|_{2}^{2}\right)\right) \quad \forall i=1, \ldots, N .
\end{aligned}\right.
$$

Since $z_{4}(x)$ is arbitrary, it can be chosen of the form $\|x\|_{2}^{2 \tilde{d}}$ with $\tilde{d} \in \mathbb{N}$ in order to ensure that the right hand sides of the inequalities in the previous condition, with the second one changed in sign, admit positive definite Gram matrices, see for instance [4]. This implies that there exists $\Phi>0$ satisfying (24) for

$$
\phi(x)=z_{1}(x)\|x\|_{2}^{2 \tilde{d}}
$$

which is a homogeneous polynomial of degree $2 d$ with

$$
d=d_{1}+\tilde{d} .
$$

Moreover, there exists $\Psi$ satisfying (38) since such a matrix can be simply chosen diagonal with positive entries on the diagonal. At this point, the proof proceeds by reverting the proof of Theorem 3 and observing that the existence of $\Theta_{1}, \ldots, \Theta_{N}$ satisfying (49) is ensured by $z_{4}(x)$.

Theorem 4 states that the LMI condition provided by Theorem 3 can be used to approximate arbitrarily well the RMS gain of (1) in the case of arbitrary switching by increasing $d$. This means that, for all $\varepsilon>0$ there exists $d$ such that the upper bound $\hat{\gamma}_{R M S}$ in (51) satisfies

$$
\gamma_{R M S} \leq \hat{\gamma}_{R M S} \leq \gamma_{R M S}+\varepsilon
$$

at least when $\Psi$ is chosen according to (38).

\section{Switching with Dwell Time Constraints}

This section addresses Problem 2, i.e., the determination of the sought performance indexes in the case of switching with dwell time constraints. Contrary to the case of arbitrary switching presented in the previous section where a Lyapunov function common to all the subsystems of (1) is searched for, here the idea is to exploit a family of Lyapunov functions mutually constrained by the dwell time specification. In this respect, it is useful to observe that such a family of Lyapunov functions is guaranteed to exist for asymptotically stable switched systems with dwell time constraints, see for instance [28].

Let us start by considering the determination of the $\mathcal{H}_{2}$ norm in the case of switching with dwell time constraints. We want to search for a family of HRLFs $v_{i}(x), i=1, \ldots, N$, of total degree $2 d$ and relative degree 2 , with common denominator. These HRLF candidates can be expressed analogously to (24) as

$$
\left\{\begin{aligned}
v_{i}(x) & =\frac{\phi_{i}(x)}{\psi(x)} \\
\phi_{i}(x) & =b(x, d)^{\prime} \Phi_{i} b(x, d) \\
\psi(x) & =b(x, d-1)^{\prime} \Psi b(x, d-1)
\end{aligned}\right.
$$

where $\Phi_{i} \in \mathbb{R}^{c(n, d) \times c(n, d)}, \Phi_{i}=\Phi_{i}^{\prime}$, and $\Psi \in$ $\mathbb{R}^{c(n, d-1) \times c(n, d-1)}, \Psi=\Psi^{\prime}$. For $s=0,1$, let $U_{i, s}$ be the matrix satisfying

$$
b\left(e^{A_{i} T} x, d-s\right)=U_{i, s} b(x, d-s) .
$$

For $\Phi_{i}$ and $\Psi$ as in (56), let us define

$$
\left\{\begin{aligned}
Q_{i, 1}\left(\Phi_{j}\right) & =J_{1}^{\prime}\left(\left(U_{i, 0}^{\prime} \Phi_{j} U_{i, 0}\right) \otimes \Psi\right) J_{1} \\
Q_{i, 2}\left(\Phi_{i}\right) & =J_{1}^{\prime}\left(\Phi_{i} \otimes\left(U_{i, 1}^{\prime} \Psi U_{i, 1}\right)\right) J_{1} \\
Q_{i, 3} & =J_{2}^{\prime}\left(\Psi \otimes\left(U_{i, 1}^{\prime} \Psi U_{i, 1}\right) \otimes \Delta_{i}\right) J_{2}
\end{aligned}\right.
$$

where

$$
\Delta_{i}=\int_{0}^{T} e^{A_{i}^{\prime} t} C_{i}^{\prime} C_{i} e^{A_{i} t} d t
$$

Let us define

$$
Q_{i}\left(\Phi_{i}, \Phi_{j}\right)=Q_{i, 1}\left(\Phi_{j}\right)-Q_{i, 2}\left(\Phi_{i}\right)+Q_{i, 3} .
$$

Theorem 5: Consider the case of switching with dwell time constraints, i.e., $\mathcal{D}=\mathcal{D}_{T}$. Let $d \geq 1$ and $\Psi>0$ be chosen. Suppose that there exist $\xi, \Phi_{i}, \Theta_{i}$ and $\Omega_{i, j}, i, j=1, \ldots, N$, $i \neq j$, satisfying the system of LMIs

$$
\left\{\begin{aligned}
0 & <\Phi_{i} \forall i=1, \ldots, N \\
0 & >E_{i}\left(\Phi_{i}\right)+M_{1}\left(\Theta_{i}\right) \forall i=1, \ldots, N \\
0 & <\xi-g_{i}\left(\Phi_{i}\right) \forall i=1, \ldots, N \\
0 & >Q_{i}\left(\Phi_{i}, \Phi_{j}\right)+M_{1}\left(\Omega_{i, j}\right) \forall i, j=1, \ldots, N, i \neq j .
\end{aligned}\right.
$$

Then, $v_{i}(x)$ in (56) is an HRLF for the $i$-th subsystem of (1), and

$$
\gamma_{\mathcal{H}_{2}}<\sqrt{\xi}
$$

Proof. Suppose that the system of LMIs (61) holds. Let us observe that the first three LMIs in this system are the LMI 
condition (33) provided by Theorem 1 for the case of arbitrary switching, with the difference that $N$ HRLFs of the form (56) are searched for instead of an HRLF $v(x)$ of the form (24). This means that $\sqrt{\xi}$ is an upper bound of the $\mathcal{H}_{2}$ norm of each subsystem of (1). In order to show that (62) holds for all switching policies in $\mathcal{D}_{T}$, let us suppose that $\sigma$ presents a jump at a generic time instant, i.e.,

$$
\left\{\begin{array}{l}
\sigma\left(\tilde{t}^{-}\right)=j \\
\sigma(t)=i \forall t \in[\tilde{t}, \tilde{t}+T) \\
\tilde{t} \in[0, \infty), i, j \in\{1, \ldots, N\}, i \neq j .
\end{array}\right.
$$

The fourth LMI in (61) implies that

$$
\begin{gathered}
v_{i}(x)>v_{j}\left(e^{A_{i} T} x\right)+x^{\prime} \Delta_{i} x \\
\forall x \neq 0_{n} \forall i, j=1, \ldots, N, i \neq j .
\end{gathered}
$$

Hence, each HRLF evaluated at a state $x$ at the time instant $t=\tilde{t}^{-}$is greater than any other HRLF evaluated at the state obtained from $x$ after a time $T$, i.e., $e^{A_{i} T} x$ at the time instant $t=\tilde{t}^{-}+T$, plus the energy during this time starting from $x$, i.e., $x^{\prime} \Delta_{i} x$. This implies that $\sqrt{\xi}$ is an upper bound of the $\mathcal{H}_{2}$ norm for all switching policies in $\mathcal{D}_{T}$.

Theorem 5 provides a sufficient LMI condition for establishing upper bounds of the $\mathcal{H}_{2}$ norm of (1) in the case of switching with dwell time constraints. The matrices used to define the LMI condition (61) can be easily built, see the Appendix for details.

The best upper bound provided by Theorem 5 for chosen $d$ and $\Psi$ can be found by minimizing $\xi$ subject to the LMIs (61), and is equal to

$$
\hat{\gamma}_{\mathcal{H}_{2}}=\sqrt{\xi_{\mathcal{H}_{2}}}
$$

where $\xi_{\mathcal{H}_{2}}$ is the solution of the SDP

$$
\begin{aligned}
\xi_{\mathcal{H}_{2}}= & \inf _{\xi, \Phi_{i}, \Theta_{i}, \Omega_{i, j}} \xi \\
& \text { s.t. }(61) \text { holds. }
\end{aligned}
$$

The LMI condition provided by Theorem 5 is not only sufficient but also necessary by using sufficiently large values of $d$. This result is a direct extension of Theorem 2 for the case of arbitrary switching and is omitted for brevity.

Next, let us consider the determination of the RMS gain in the case of switching with dwell time constraints. Let us express $\phi_{i}(x)$ in (56) as

$$
\phi_{i}(x)=x^{\prime} Y\left(x, \Phi_{i}\right) x
$$

where $Y\left(x, \Phi_{i}\right)$ is a symmetric matrix homogeneous polynomial of degree $2 d-2$ depending on $\Phi_{i}$. Let us introduce the set

$$
\mathcal{Z}=\left\{Z(x)=Z(x)^{\prime}: Z(x)\right. \text { is a matrix homogeneous }
$$
polynomial of degree $2 d-2$ such that $x^{\prime} Z(x) x=0$ for all $\left.x\right\}$

and let $Z(x, \Upsilon)$ be a linear parametrization of $\mathcal{Z}$ where $\Upsilon$ is a free vector. Let us define

$$
\Xi\left(x, \Phi_{i}, \Upsilon_{i}\right)=Y\left(x, \Phi_{i}\right)+Z\left(x, \Upsilon_{i}\right)
$$

where $\Upsilon_{i}$ is a free vector. Let us define

$$
\begin{gathered}
V_{i}\left(x, \Lambda_{1}, \Lambda_{2}\right)= \\
\left(\begin{array}{c}
e^{H_{i}^{\prime} T} \Lambda_{2} \psi(x) e^{H_{i} T}-\Lambda_{1} \psi\left(e^{H_{i} T} x\right)+R_{i} \psi(x) \psi\left(e^{H_{i} T} x\right) \\
\star \\
e^{H_{i}^{\prime} T}\left(\Lambda_{2} \psi(x)-P_{i} \psi(x) \psi\left(e^{H_{i} T} x\right)\right) \\
\Lambda_{2} \psi(x)-\left(P_{i}+g^{2} S_{i}^{-1}\right) \psi(x) \psi\left(e^{H_{i} T} x\right)
\end{array}\right)
\end{gathered}
$$

where $\Lambda_{1}, \Lambda_{2}$ are symmetric matrices, $g \in \mathbb{R}, P_{i}$ is the solution of the algebraic Riccati equation (ARE)

$$
\begin{aligned}
0= & A_{i}^{\prime} P_{i}+P_{i} A_{i}+C_{i}^{\prime} C_{i}+\left(P_{i} B_{i}+C_{i}^{\prime} D\right) \\
& \cdot\left(g^{2} I-D_{i}^{\prime} D_{i}\right)^{-1}\left(P_{i} B_{i}+C_{i}^{\prime} D\right)^{\prime},
\end{aligned}
$$

and

$$
\left\{\begin{aligned}
R_{i} & =P_{i}-e^{H_{i}^{\prime} T} P_{i} e^{H_{i} T} \\
S_{i} & =\int_{0}^{\infty} e^{H_{i} t} B_{i}\left(I-g^{-2} D_{i}^{\prime} D_{i}\right)^{-1} B_{i}^{\prime} e^{H_{i}^{\prime} t} d t \\
H_{i} & =A_{i}+B_{i} L_{i} \\
L_{i} & =\left(g^{2} I-D_{i}^{\prime} D_{i}\right)^{-1}\left(P_{i} B_{i}+C_{i}^{\prime} D\right)^{\prime} .
\end{aligned}\right.
$$

It is useful to remark that the ARE (69) admits a unique positive definite solution $P_{i}$ whenever the pair $\left(A_{i}, B_{i}\right)$ is reachable, the pair $\left(A_{i}, C_{i}\right)$ is observable, and $g$ is greater than the RMS gain of the $i$-th subsystem of (1), see for instance [10] for details. Let us express $V_{i}\left(x, \Xi\left(x, \Phi_{i}, \Upsilon_{i}\right), \Xi\left(x, \Phi_{j}, \Upsilon_{j}\right)\right)$ as

$$
\begin{gathered}
V_{i}\left(x, \Xi\left(x, \Phi_{i}, \Upsilon_{i}\right), \Xi\left(x, \Phi_{j}, \Upsilon_{j}\right)\right)=(b(x, 2 d-2) \otimes I)^{\prime} \\
\cdot W_{i}\left(\Phi_{i}, \Phi_{j}, \Upsilon_{i}, \Upsilon_{j}\right)(b(x, 2 d-2) \otimes I)
\end{gathered}
$$

for a symmetric matrix $W_{i}\left(\Phi_{i}, \Phi_{j}, \Upsilon_{i}, \Upsilon_{j}\right)$. Let $M_{3}(\cdot)$ be a linear parametrization of the set

$$
\begin{aligned}
\mathcal{M}_{3}= & \left\{M=M^{\prime}:(b(x, 2 d-2) \otimes I)^{\prime} M\right. \\
& \cdot(b(x, 2 d-2) \otimes I)=0 \forall x\} .
\end{aligned}
$$

Theorem 6: Consider the case of switching with dwell time constraints, i.e., $\mathcal{D}=\mathcal{D}_{T}$. Let $d \geq 1, \Psi>0$ and $g>0$ be chosen. Suppose that there exist $\Phi_{i}, \Theta_{i}, \Omega_{i, j}$ and $\Upsilon_{i}, i, j=$ $1, \ldots, N, i \neq j$, satisfying the system of LMIs

$$
\left\{\begin{aligned}
0< & \Phi_{i} \forall i=1, \ldots, N \\
0> & F_{i}\left(\Phi_{i}, g^{2}\right)+G_{i}+M_{2}\left(\Theta_{i}\right) \forall i=1, \ldots, N \\
0> & W_{i}\left(\Phi_{i}, \Phi_{j}, \Upsilon_{i}, \Upsilon_{j}\right)+M_{3}\left(\Omega_{i, j}\right) \\
& \forall i, j=1, \ldots, N, i \neq j .
\end{aligned}\right.
$$

Then, $v_{i}(x)$ in (56) is an HRLF for the $i$-th subsystem of (1), and

$\gamma_{R M S}<g$

Proof. Suppose that the system of LMIs (73) holds. Let us observe that the first two LMIs in this system are the LMI condition (49) provided by Theorem 3 for the case of arbitrary switching, with the difference that $\xi$ is replaced by $g^{2}$, and $N$ HRLFs of the form (56) are searched for instead of an HRLF $v(x)$ of the form (24). This means that $g$ is an upper bound of the RMS gain of each subsystem of (1). In order to show 
that (74) holds for all switching policies in $\mathcal{D}_{T}$, let us suppose that $\sigma$ presents a jump at a generic time instant, i.e.,

$$
\left\{\begin{array}{l}
\sigma\left(\tilde{t}^{-}\right)=j \\
\sigma(t)=i \forall t \in[\tilde{t}, \tilde{t}+T) \\
\tilde{t} \in[0, \infty), i, j \in\{1, \ldots, N\}, i \neq j .
\end{array}\right.
$$

The third LMI in (73) implies that

$$
\begin{gathered}
V_{i}\left(x, \Xi\left(x, \Phi_{i}, \Upsilon_{i}\right), \Xi\left(x, \Phi_{j}, \Upsilon_{j}\right)\right)<0 \\
\forall x \neq 0_{n} \forall i, j=1, \ldots, N, i \neq j .
\end{gathered}
$$

Proceeding analogously to [13], one obtains that

$$
\begin{gathered}
v_{i}(x)>\sup _{w(\cdot)}\left(v_{j}\left(e^{A_{i} T} x\right)+\int_{\tilde{t}}^{\tilde{t}+T}\left(\|y\|_{2}^{2}-g^{2}\|w\|_{2}^{2}\right) d t\right) \\
\forall x \neq 0_{n} \forall i, j=1, \ldots, N, i \neq j .
\end{gathered}
$$

Hence, each HRLF evaluated at a state $x$ at the time instant $t=\tilde{t}^{-}$is greater than the worst case, over all possible inputs $w$, of any other HRLF evaluated at the state obtained from $x$ after a time $T$, i.e., $e^{A_{i} T} x$ at the time instant $t=\tilde{t}^{-}+T$, plus the dissipated energy during this time starting from $x$, i.e., the integral between $\tilde{t}$ and $\tilde{t}+T$ of $\|y\|_{2}^{2}-g^{2}\|w\|_{2}^{2}$. This implies that $g$ is an upper bound of the RMS gain for all switching policies in $\mathcal{D}_{T}$.

Theorem 6 provides a sufficient LMI condition for establishing upper bounds of the RMS gain of (1) in the case of switching with dwell time constraints. The matrices used to define the LMI condition (73) can be easily built, see the Appendix for details.

The best upper bound provided by Theorem 6 for chosen $d$ and $\Psi$ can be found by minimizing $g$ subject to the LMIs (73), and is equal to

$$
\begin{aligned}
\hat{\gamma}_{R M S}= & \inf _{g, \Phi_{i}, \Theta_{i}, \Omega_{i, j}, \Upsilon_{i}} g \\
& \text { s.t. (73) holds. }
\end{aligned}
$$

Contrary to the upper bounds introduced in the previous sections, $\hat{\gamma}_{R M S}$ in (75) cannot be found by solving an SDP. Indeed, the LMIs (73) are nonlinear in $g$. Nevertheless, one can solve (75) through a bisection search on $g$ where the feasibility of these LMIs is tested at each step.

Also, contrary to the LMI conditions provided by Theorems 1, 3 and 5, which are not only sufficient but also necessary, the LMI condition provided by Theorem 5 could be sufficient only. Nevertheless, this LMI condition is not more conservative than the existing LMI conditions based on quadratic Lyapunov functions, which are recovered by Theorem 5 with $d=1$. Moreover, as shown by Example 4 in the next section, the LMI condition provided by Theorem 5 can significantly improve the results obtained by the existing LMI conditions.

\section{EXAMPLES}

In this section we present some numerical examples. The LMI feasibility tests and the SDPs are solved with the toolbox SeDuMi [26] for Matlab on a personal computer with Windows 10, Intel Core i7, $3.4 \mathrm{GHz}, 8$ GB RAM. The vector $b(x, d)$ is chosen according to (10). The matrix $\Psi$ is chosen as the diagonal matrix that satisfies (38) (unless specified otherwise).

\section{A. Example 1}

Let us consider (1) with $N=2$ and

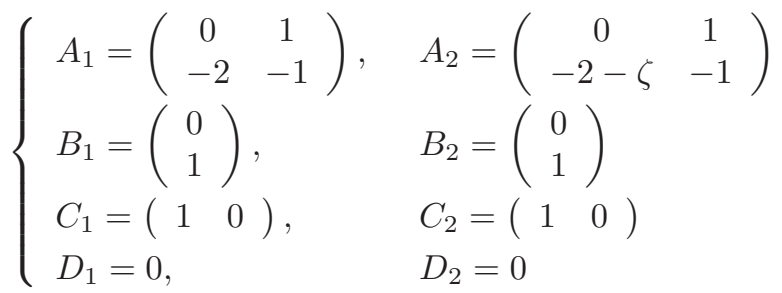

where $\zeta \in \mathbb{R}$ is a parameter. The problem is to determine the $\mathcal{H}_{2}$ norm in the case of arbitrary switching, i.e., the index $\gamma_{\mathcal{H}_{2}}$ in (3) for $\mathcal{D}=\mathcal{D}_{a r b}$.

We start by considering the case $\zeta=3$. By using $d=1$ (in this case HRLFs are quadratic Lyapunov functions), the upper bound in (35) is

$$
\hat{\gamma}_{\mathcal{H}_{2}}=0.953 \text {. }
$$

The SDP (36) has 4 LMI scalar variables, and its computational time is less than one second. The found HRLF is as in (24) with

$$
\left\{\begin{array}{l}
\phi(x)=3.278 x_{1}^{2}+1.074 x_{1} x_{2}+0.909 x_{2}^{2} \\
\psi(x)=1 .
\end{array}\right.
$$

Some of the matrices in the SDP (36) are as follows:

$$
\left\{\begin{array}{c}
A_{1,0}=A_{1}, A_{2,0}=A_{2}, A_{1,1}=0, A_{2,1}=0 \\
\Psi=1, b(x, d)=x, M_{1}(\theta)=0_{2 \times 2} \\
J_{1}=I_{2}, J_{2}=I_{2} .
\end{array}\right.
$$

By using $d=2$, the upper bound in (35) is

$$
\hat{\gamma}_{\mathcal{H}_{2}}=0.689 \text {. }
$$

The SDP (36) has 13 LMI scalar variables, and its computational time is less than one second. The found HRLF is as in (24) with

$$
\left\{\begin{aligned}
\phi(x)= & 1.624 x_{1}^{4}+0.502 x_{1}^{3} x_{2}+2.353 x_{1}^{2} x_{2}^{2} \\
& +0.669 x_{1} x_{2}^{3}+0.474 x_{2}^{4} \\
\psi(x)= & x_{1}^{2}+x_{2}^{2} .
\end{aligned}\right.
$$


Some of the matrices in the SDP (36) are as follows:

$$
\begin{aligned}
& A_{1,0}=\left(\begin{array}{ccc}
0 & 2 & 0 \\
-2 & -1 & 1 \\
0 & -4 & -2
\end{array}\right), A_{2,0}=\left(\begin{array}{ccc}
0 & 2 & 0 \\
-5 & -1 & 1 \\
0 & -10 & -2
\end{array}\right) \\
& A_{1,1}=A_{1}, A_{2,1}=A_{2}, \Psi=I_{2}, b(x, d)=\left(\begin{array}{c}
x_{1}^{2} \\
x_{1} x_{2} \\
x_{2}^{2}
\end{array}\right) \\
& M_{1}(\theta)=\left(\begin{array}{cccc}
0 & 0 & -\theta_{1} & -\theta_{2} \\
\star & 2 \theta_{1} & \theta_{2} & -\theta_{3} \\
\star & \star & 2 \theta_{3} & 0 \\
\star & \star & \star & 0
\end{array}\right) . \\
& J_{1}=\left(\begin{array}{llll}
1 & 0 & 0 & 0 \\
0 & 1 & 0 & 0 \\
0 & 1 & 0 & 0 \\
0 & 0 & 1 & 0 \\
0 & 0 & 1 & 0 \\
0 & 0 & 0 & 1
\end{array}\right), J_{2}=\left(\begin{array}{llll}
1 & 0 & 0 & 0 \\
0 & 1 & 0 & 0 \\
0 & 1 & 0 & 0 \\
0 & 0 & 1 & 0 \\
0 & 1 & 0 & 0 \\
0 & 0 & 1 & 0 \\
0 & 0 & 1 & 0 \\
0 & 0 & 0 & 1
\end{array}\right) .
\end{aligned}
$$

Lastly, by using $d=3$, the upper bound in (35) is

$$
\hat{\gamma}_{\mathcal{H}_{2}}=0.631 \text {. }
$$

The SDP (36) has 31 LMI scalar variables, and its computational time is less than one second. The found HRLF is as in (24) with

$$
\left\{\begin{aligned}
\phi(x)= & 1.397 x_{1}^{6}+0.502 x_{1}^{5} x_{2}+3.384 x_{1}^{4} x_{2}^{2}+0.898 x_{1}^{3} x_{2}^{3} \\
& +2.404 x_{1}^{2} x_{2}^{4}+0.625 x_{1} x_{2}^{5}+0.398 x_{2}^{6} \\
\psi(x)= & \left(x_{1}^{2}+x_{2}^{2}\right)^{2} .
\end{aligned}\right.
$$

It is interesting to observe that, though non-conservatism is ensured by Theorem 2 for sufficiently large values of $d$ with $\Psi$ satisfying (38), the results obtained for fixed values of $d$ might be improved by using different choices of $\Psi$. Indeed, by using for $d=3$ the choice

$$
\Psi=\operatorname{diag}(4,7,1)
$$

we obtain the new upper bound

$$
\hat{\gamma}_{\mathcal{H}_{2}}=0.608 \text {. }
$$

Next, we repeat the previous computations for different values of $\zeta$ (with $\Psi$ satisfying (38)). Fig. 1 shows the found upper bounds.

\section{B. Example 2}

Let us consider (1) with $N=2$ and

$$
\begin{aligned}
& A_{1}=\left(\begin{array}{ccc}
1 & 3 & 1 \\
-1 & -2 & -1 \\
-1 & -2 & -2
\end{array}\right), \quad A_{2}=\left(\begin{array}{ccc}
2 & 3 & 2 \\
-2 & -2 & -1 \\
-2 & -2 & -2
\end{array}\right) \\
& B_{1}=\left(\begin{array}{l}
1 \\
0 \\
0
\end{array}\right), \quad B_{2}=\left(\begin{array}{c}
0 \\
-1 \\
0
\end{array}\right) \\
& C_{1}=\left(\begin{array}{ccc}
1 & 0 & 0 \\
0 & 1 & 0
\end{array}\right), \quad C_{2}=\left(\begin{array}{ccc}
1 & 0 & 0 \\
0 & 1 & 0
\end{array}\right) \\
& D_{1}=\left(\begin{array}{l}
1 \\
0
\end{array}\right), \quad D_{2}=\left(\begin{array}{l}
1 \\
0
\end{array}\right) \text {. }
\end{aligned}
$$

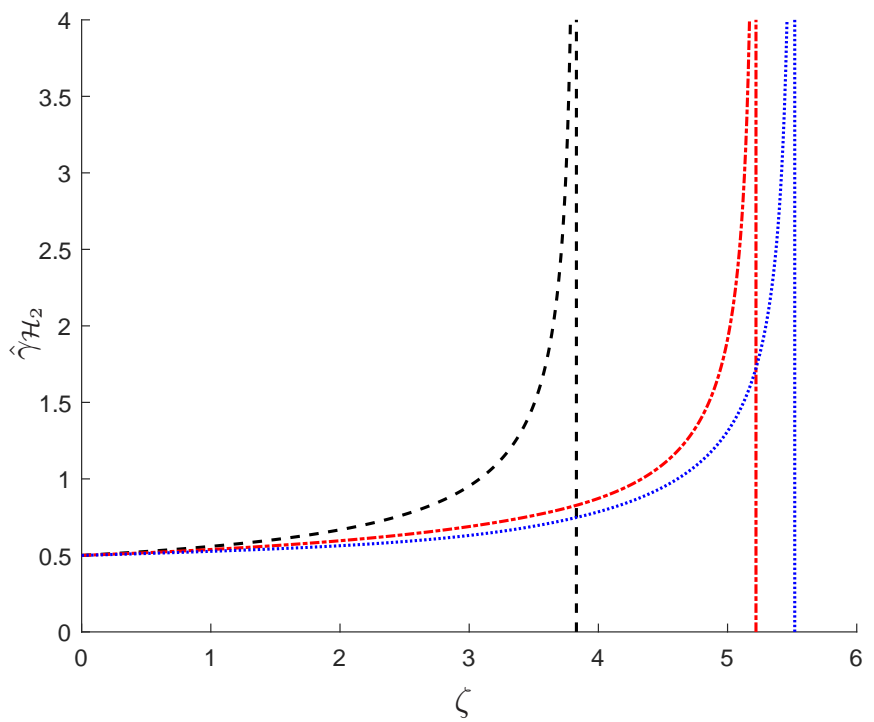

Fig. 1. Example 1. Upper bound $\hat{\gamma}_{\mathcal{H}}$ for $d=1$ (dashed black curve), $d=2$ (dashdot red curve) and $d=3$ (dotted blue curve) versus $\zeta$. The vertical lines show the largest value of $\zeta$ for which the upper bound is finite.

The problem is to determine the RMS gain in the case of arbitrary switching, i.e., the index $\gamma_{R M S}$ in (5) for $\mathcal{D}=\mathcal{D}_{\text {arb }}$.

By using $d=1,2,3$, the upper bound in (51) is as follows:

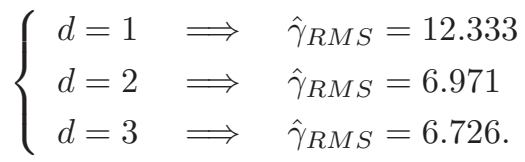

The numbers of LMI scalar variables in the SDP (52) are, respectively, 7, 166 and 1056 . The computational times required for solving the SDP are, respectively, 0.5, 0.6 and 4.7 seconds. The upper bound $\hat{\gamma}_{R M S}$ found with $d=1$ coincides with the upper bound provided by [13].

\section{Example 3}

Let us consider (1) with $N=3$ and

$$
\begin{cases}A_{1}=\left(\begin{array}{cc}
0 & 1 \\
-2 & -1
\end{array}\right), & A_{2}=\left(\begin{array}{cc}
0 & 1 \\
-12 & -1
\end{array}\right) \\
B_{1}=\left(\begin{array}{l}
0 \\
1
\end{array}\right), & B_{2}=\left(\begin{array}{l}
0 \\
1
\end{array}\right) \\
C_{1}=\left(\begin{array}{ll}
1 & 0
\end{array}\right), & C_{2}=\left(\begin{array}{ll}
0 & 1
\end{array}\right) . \\
D_{1}=0, & D_{2}=0 \\
A_{3}=\left(\begin{array}{cc}
0 & 1 \\
-2 & -0.1
\end{array}\right), & B_{3}=\left(\begin{array}{c}
0 \\
-1
\end{array}\right) \\
C_{3}=\left(\begin{array}{ll}
0 & 1
\end{array}\right), & D_{3}=0 .\end{cases}
$$

The problem is to determine the $\mathcal{H}_{2}$ norm in the case of switching with dwell time constraints, i.e., the index $\gamma_{\mathcal{H}_{2}}$ in (3) for $\mathcal{D}=\mathcal{D}_{T}$.

We start by considering the case $T=1.6$. By using $d=1$, the upper bound in (63) is

$$
\hat{\gamma}_{\mathcal{H}_{2}}=4.208 \text {. }
$$


The SDP (64) has 10 LMI scalar variables, and its computational time is less than one second. The found HRLF is as in (24) with

$$
\left\{\begin{array}{l}
\phi(x)=36.716 x_{1}^{2}+0.377 x_{1} x_{2}+17.710 x_{2}^{2} \\
\psi(x)=1 .
\end{array}\right.
$$

By using $d=2$, the upper bound in (63) is

$$
\hat{\gamma}_{\mathcal{H}_{2}}=3.073 \text {. }
$$

The SDP (64) has 46 LMI scalar variables, and its computational time is less than one second. The found HRLF is as in (24) with

$$
\left\{\begin{aligned}
\phi(x)= & 19.517 x_{1}^{4}+0.013 x_{1}^{3} x_{2}+29.389 x_{1}^{2} x_{2}^{2} \\
& +0.144 x_{1} x_{2}^{3}+9.445 x_{2}^{4} \\
\psi(x)= & x_{1}^{2}+x_{2}^{2}
\end{aligned}\right.
$$

Lastly, by using $d=3$, the upper bound in (63) is

$$
\hat{\gamma}_{\mathcal{H}_{2}}=2.114
$$

The SDP (64) has 121 LMI scalar variables, and its computational time is less than one second. The found HRLF is as in (24) with

$$
\left\{\begin{aligned}
\phi(x)= & 15.252 x_{1}^{6}+0.04 x_{1}^{5} x_{2}+38.12 x_{1}^{4} x_{2}^{2}-0.192 x_{1}^{3} x_{2}^{3} \\
& +30.584 x_{1}^{2} x_{2}^{4}+0.062 x_{1} x_{2}^{5}+7.425 x_{2}^{6} \\
\psi(x)= & \left(x_{1}^{2}+x_{2}^{2}\right)^{2} .
\end{aligned}\right.
$$

Next, we repeat the previous computations for different values of $T$. Fig. 2 shows the found upper bounds.

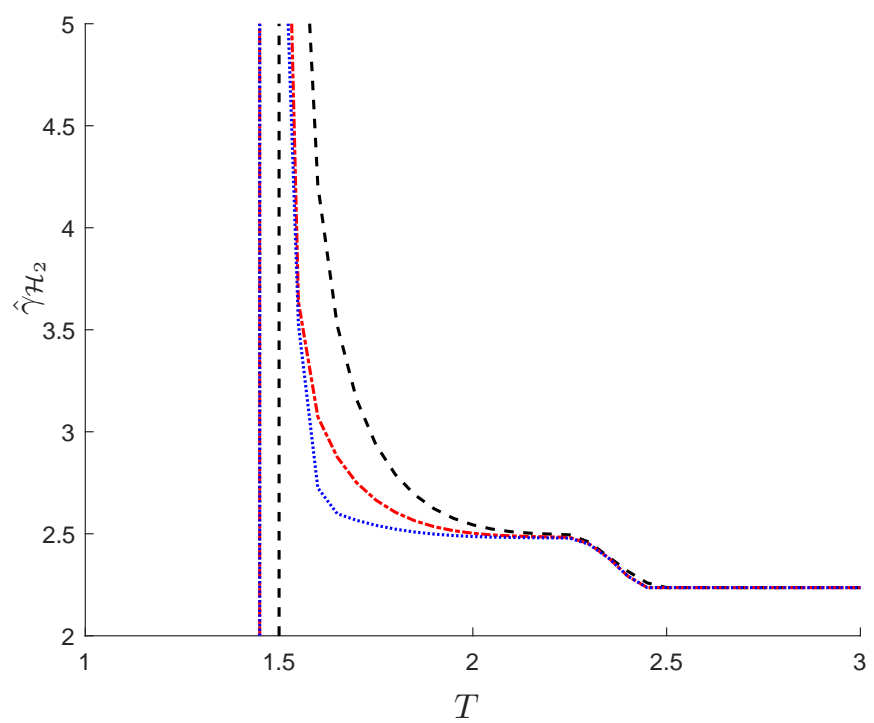

Fig. 2. Example 3. Upper bound $\hat{\gamma}_{\mathcal{H}_{2}}$ for $d=1$ (dashed black curve), $d=2$ (dashdot red curve) and $d=3$ (dotted blue curve) versus $T$. The vertical lines show the smallest value of $T$ for which the upper bound is finite.

\section{Example 4}

Let us consider (1) with $N=2$ and

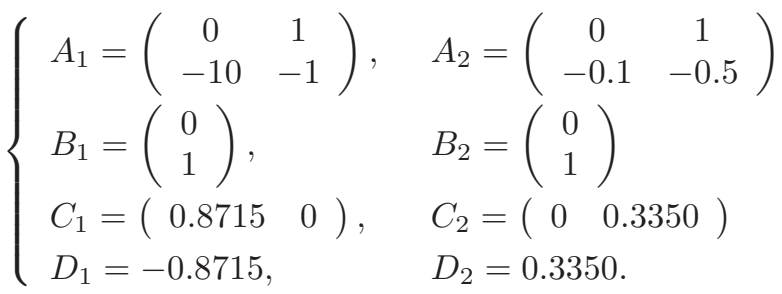

This system has been considered in [13]. Here we consider the problem to determine the RMS gain in the case of switching with dwell time constraints, i.e., the index $\gamma_{R M S}$ in (5) for $\mathcal{D}=\mathcal{D}_{T}$ and $T=3$.

By using $d=1,2,3$, the upper bound in (75) is as follows:

$$
\left\{\begin{array}{lll}
d=1 & \Longrightarrow & \hat{\gamma}_{R M S}=12.473 \\
d=2 & \Longrightarrow & \hat{\gamma}_{R M S}=9.769 \\
d=3 & \Longrightarrow & \hat{\gamma}_{R M S}=8.767 .
\end{array}\right.
$$

The numbers of LMI scalar variables in the LMI condition (73) are, respectively, 7, 96 and 349. The computational times required for solving the SDP are less than 1 second. The upper bound $\hat{\gamma}_{R M S}$ found with $d=1$ coincides with the upper bound provided by [13].

\section{CONCLUSIONS}

This paper has addressed the problems of determining two key performance indexes of continuous-time switched linear systems, specifically the $\mathcal{H}_{2}$ norm and the RMS gain. A novel class of Lyapunov functions has been proposed for reaching this goal, called HRLFs. It has been shown that sufficient conditions for establishing upper bounds of the sought performance indexes in the case of arbitrary switching can be given in terms of LMI feasibility tests by searching for an HRLF of chosen degree. Moreover, it has been shown that these conditions are also necessary by searching for an HRLF of degree sufficiently large. Hence, the case of switching with dwell time constraints has been considered, showing that analogous LMI conditions can be obtained by searching for a family of HRLFs mutually constrained by the dwell time specification.

Several directions can be investigated in future work. One of these is the extension of the proposed methodology to the case of discrete-time switched linear systems. Another direction concerns the design of feedback controllers for ensuring desired performance indexes.

\section{ACKNOWLEDGEMENTS}

The authors would like to thank the Associate Editor and the Reviewers for their useful comments.

\section{APPENDIX}

Preliminaries:

- $b(x, d)$ is supposed chosen according to $(10)$;

- let $K \in \mathbb{N}^{c(n, d) \times n}$ be the matrix whose $(i, j)$-th entry is the power of $x_{j}$ in the $i$-th entry of $b(x, d)$; 
- for $x \in \mathbb{R}^{n}$ and $k \in \mathbb{N}$, define the functions

$$
\left\{\begin{aligned}
\nu\left(x^{k}\right) & =\sum_{i=1}^{n} \sum_{j=k_{i}+1}^{\mu_{i}(k)} c\left(n-i, \mu_{i}(k)-j\right) \\
\mu_{i}(k) & =\sum_{j=i}^{n} k_{j}
\end{aligned}\right.
$$

- for $Q \in \mathbb{R}^{n \times n}$, let $\operatorname{vec}(Q) \in \mathbb{R}^{n^{2}}$ contain the entries of $Q$, and let mat $(\cdot)$ satisfy $\operatorname{mat}(\operatorname{vec}(Q))=Q$;

- for $Q \in \mathbb{R}^{n \times n}, Q=Q^{\prime}$, let $\operatorname{vec}_{\mathrm{s}}(Q) \in \mathbb{R}^{n(n+1) / 2}$ contain the independent entries of $Q$, and let $\operatorname{mat}_{\mathrm{S}}(\cdot)$ satisfy $\operatorname{mat}_{\mathrm{s}}\left(\operatorname{vec}_{\mathrm{s}}(Q)\right)=Q$.

The matrix $A_{i, s}$ in (26) can be built as follows:

1) let $A_{i, s}$ be a $c(n, d-s) \times c(n, d-s)$ null matrix;

2) for all $j=1, \ldots, c(n, d-s)$ and $k, l=1, \ldots, n$

a) let $a$ be the $j$-th row of $K$;

b) if $a_{k}>0$

i) set $\tilde{a}=a$, add -1 to $\tilde{a}_{k}$, and add 1 to $\tilde{a}_{l}$;

ii) set $f=\nu(\tilde{a})$;

iii) add $a_{k}\left(A_{i}\right)_{k, l}$ to $\left(A_{i, s}\right)_{j, f}$.

The matrix $B_{i, s}$ in (42) can be built as follows:

1) let $B_{i, s}$ be a $c(n, d-s) \times c(n, d-s-1) m$ null matrix;

2) for all $j=1, \ldots, c(n, d-s), k=1, \ldots, n$ and $l=$ $1, \ldots, m$

a) let $a$ be the $j$-th row of $K$;

b) if $a_{k}>0$

i) set $\tilde{a}=a$, and add -1 to $\tilde{a}_{k}$;

ii) set $f=(\nu(\tilde{a})-1) m+l$;

iii) add $a_{k}\left(B_{i}\right)_{k, l}$ to $\left(B_{i, s}\right)_{j, f}$.

The matrix $J_{1}$ in (28) is the $c(n, d) c(n, d-1) \times c(n, 2 d-1)$ matrix whose nonzero entries are 1 in the positions $(i, j)$

$$
\left\{\begin{array}{l}
i=\left(q_{1}-1\right) c(n, d-1)+q_{2} \\
j=\nu\left((b(x, d))_{q_{1}}(b(x, d-1))_{q_{2}}\right) \\
q_{1}=1, \ldots, c(n, d), q_{2}=1, \ldots, c(n, d-1) .
\end{array}\right.
$$

The matrix $J_{2}$ in (28) is the $c(n, d-1)^{2} n \times c(n, 2 d-1)$ matrix whose nonzero entries are 1 in the positions $(i, j)$

$$
\left\{\begin{array}{l}
i=\left(\left(q_{1}-1\right) c(n, d-1)+q_{2}-1\right) n+q_{3} \\
j=\nu\left((b(x, d-1))_{q_{1}}(b(x, d-1))_{q_{2}} x_{q_{3}}\right) \\
q_{1}, q_{2}=1, \ldots, c(n, d-1), q_{3}=1, \ldots, n
\end{array}\right.
$$

The matrix $J_{3}$ in (44) is the $c(n, d-1)^{2} m \times c(n, 2 d-2) m$ matrix whose nonzero entries are 1 in the positions $(i, j)$

$$
\left\{\begin{array}{l}
i=\left(\left(q_{1}-1\right) m+q_{2}-1\right) c(n, d-1)+q_{3} \\
j=\left(\nu\left((b(x, d-1))_{q_{1}}(b(x, d-1))_{q_{3}}\right)-1\right) m+q_{2} \\
q_{1}, q_{3}=1, \ldots, c(n, d-1), q_{2}=1, \ldots, m .
\end{array}\right.
$$

The matrix $J_{4}$ in (44) is the $c(n, d) c(n, d-2) m \times c(n, 2 d-$
2) $m$ matrix whose nonzero entries are 1 in the positions $(i, j)$

$$
\left\{\begin{array}{l}
i=\left(\left(q_{1}-1\right) c(n, d-2)+q_{2}-1\right) m+q_{3} \\
j=\left(\nu\left((b(x, d))_{q_{1}}(b(x, d-2))_{q_{2}}\right)-1\right) m+q_{3} \\
q_{1}=1, \ldots, c(n, d), q_{2}=1, \ldots, c(n, d-2) \\
q_{3}=1, \ldots, m .
\end{array}\right.
$$

The matrix $J_{5}$ in (44) is the $c(n, d-1)^{2} m \times c(n, 2 d-2) m$ matrix whose nonzero entries are 1 in the positions $(i, j)$

$$
\left\{\begin{array}{l}
i=\left(\left(q_{1}-1\right) c(n, d-1)+q_{2}-1\right) m+q_{3} \\
j=\left(\nu\left((b(x, d-1))_{q_{1}}(b(x, d-1))_{q_{2}}\right)-1\right) m+q_{3} \\
q_{1}, q_{2}=1, \ldots, c(n, d-1), q_{3}=1, \ldots, m
\end{array}\right.
$$

A linear parametrization $M_{1}(\Theta)$ of the set $\mathcal{M}_{1}$ in (32) can be built as follows:

1) let $T_{1}$ be the matrix satisfying $b(x, 2 d-1)^{\prime} M b(x, 2 d-1)=\tilde{b}(x)^{\prime} T_{1} \operatorname{vec}_{\mathrm{s}}(M)$ where $\tilde{b}(x)$ is a vector of distinct monomials in $x$;

2) let $T_{2}$ be a matrix whose columns span the right null space of $T_{1}$;

3) $M_{1}(\Theta)$ is given by $\operatorname{mat}_{\mathrm{s}}\left(T_{2} \Theta\right)$.

A linear parametrization $M_{2}(\Theta)$ of the set $\mathcal{M}_{2}$ in (48) can be built as follows:

1) let $T_{1}$ be the matrix satisfying $r(x, d, w)^{\prime} \operatorname{Mr}(x, d, w)=$ $\tilde{b}(x, w)^{\prime} T_{1} \operatorname{vec}_{\mathrm{s}}(M)$ where $\tilde{b}(x, w)$ is a vector of distinct monomials in $\left(x^{\prime}, w^{\prime}\right)^{\prime}$;

2) let $T_{2}$ be a matrix whose columns span the right null space of $T_{1}$

3) $M_{2}(\Theta)$ is given by $\operatorname{mat}_{\mathrm{s}}\left(T_{2} \Theta\right)$.

A matrix $Y\left(x, \Phi_{i}\right)$ satisfying (65) and a linear parametrization $Z(x, \Upsilon)$ of the set $\mathcal{Z}$ in (66) can be built as follows:

1) let $T_{1}$ and $T_{2}$ be the vectors satisfying $\quad b(x, d)^{\prime} \Phi_{i} b(x, d)=\tilde{b}(x)^{\prime} T_{1} \quad$ and $x^{\prime} \operatorname{mat}_{\mathrm{s}}\left(T_{3} b(x, 2 d-2)\right) x=\tilde{b}(x)^{\prime} T_{2}$ where $T_{3}$ is a free matrix and $\tilde{b}(x)$ is a vector of distinct monomials in $x$;

2) let $T_{4}$ be the matrix such that $T_{2}=T_{4} \operatorname{vec}\left(T_{3}\right)$;

3) let $T_{5}$ be a vector such that $T_{4} T_{5}=T_{1}$;

4) let $T_{6}$ be a matrix whose columns span the right null space of $T_{4}$;

5) $Y\left(x, \Phi_{i}\right)$ is given by $\operatorname{mat}_{\mathrm{s}}\left(\operatorname{mat}\left(T_{5}\right) b(x, 2 d-2)\right)$;

6) $Z(x, \Upsilon)$ is given by $\operatorname{mat}_{\mathrm{s}}\left(\operatorname{mat}\left(T_{6} \Upsilon\right) b(x, 2 d-2)\right)$.

A matrix $W_{i}\left(\Phi_{i}, \Phi_{j}, \Upsilon_{i}, \Upsilon_{j}\right)$ satisfying (71) and a linear parametrization $M_{3}(\Theta)$ of the set $\mathcal{M}_{3}$ in (72) can be built as follows:

1) let $T_{1}$ be the vector satisfying $q^{\prime} V_{i}\left(x, \Xi\left(x, \Phi_{i}, \Upsilon_{i}\right), \Xi\left(x, \Phi_{j}, \Upsilon_{j}\right)\right) q=\tilde{b}(x)^{\prime} T_{1}$ and let $T_{2}$ be the matrix satisfying $q^{\prime}(b(x, 2 d-2) \otimes I)^{\prime} M(b(x, 2 d-2) \otimes I) q=$ $\tilde{b}(x)^{\prime} T_{2} \operatorname{vec}_{\mathrm{S}}(M)$ where $q \in \mathbb{R}^{2 n}$ and $\tilde{b}(x)$ is a vector of distinct monomials in $x$

2) let $T_{3}$ be a vector such that $T_{2} T_{3}=T_{1}$;

3) let $T_{4}$ be a matrix whose columns span the right null space of $T_{2}$; 
4) $W_{i}\left(\Phi_{i}, \Phi_{j}, \Upsilon_{i}, \Upsilon_{j}\right)$ is given by $\operatorname{mat}_{\mathrm{s}}\left(T_{3}\right)$;

5) $M_{3}(\Theta)$ is given by $\operatorname{mat}_{\mathrm{s}}\left(T_{4} \Theta\right)$.

\section{REFERENCES}

[1] F. Blanchini and S. Miani. Set-Theoretic Methods in Control. Birkhauser, 2008.

[2] S. Boyd, L. El Ghaoui, E. Feron, and V. Balakrishnan. Linear Matrix Inequalities in System and Control Theory. SIAM, 1994.

[3] M. S. Branicky. Multiple Lyapunov functions and other analysis tools for switched and hybrid systems. IEEE Transactions on Automatic Control, 43:475-482, 1998.

[4] G. Chesi. LMI techniques for optimization over polynomials in control: a survey. IEEE Transactions on Automatic Control, 55(11):2500-2510, 2010.

[5] G. Chesi. LMI conditions for time-varying uncertain systems can be non-conservative. Automatica, 47(3):621-624, 2011.

[6] G. Chesi and P. Colaneri. On the H-two norm of switched systems via homogeneous rational Lyapunov functions. In IEEE Multi-Conference on Systems and Control, pages 667-672, Buenos Aires, Argentina, 2016.

[7] G. Chesi and P. Colaneri. On the RMS gain of switched systems via homogeneous rational Lyapunov functions. In IEEE Conference on Decision and Control, pages 7240-7245, Las Vegas, USA, 2016.

[8] G. Chesi, P. Colaneri, J. C. Geromel, R. H. Middleton, and R. Shorten. A nonconservative LMI condition for stability of switched systems with guaranteed dwell time. IEEE Transactions on Automatic Control, 57(5):1297-1302, 2012.

[9] G. Chesi, A. Garulli, A. Tesi, and A. Vicino. Homogeneous Lyapunov functions for systems with structured uncertainties. Automatica, 39(6):1027-1035, 2003.

[10] P. Colaneri, J. C. Geromel, and A. Locatelli. Control Theory and Design - An $\mathcal{R H}_{2}$ and $\mathcal{R H}_{\infty}$ Viewpoint. Academic Press, 1997.

[11] R. A. DeCarlo, M. S. Branicky, S. Pettersson, and B. Lennartson. Perspectives and results on the stability and stabilizability of hybrid systems. Proc. IEEE, 88(7):1069-1082, 2000.

[12] J. C. Geromel and P. Colaneri. Robust stability of time varying polytopic systems. Systems and Control Letters, 55(1):81-85, 2006.

[13] J. C. Geromel and P. Colaneri. $\mathcal{H}_{\infty}$ and dwell time specifications of continuous-time switched linear systems. IEEE Transactions on Automatic Control, 55(1):207-212, 2010.

[14] J. C. Geromel, P. Colaneri, and P. Bolzern. Dynamic output feedback control of switched linear systems. IEEE Transactions on Automatic Control, 53(3):720-733, 2008.

[15] J. P. Hespanha. $\mathcal{L}_{2}$-induced gains of switched linear systems. In V. D. Blondel and A. Megretski, editors, Unsolved Problems in Mathematical Systems and Control Theory, pages 131-133. Princeton University Press, 2003.

[16] J. P. Hespanha. Root-mean-square gains of switched linear systems. IEEE Transactions on Automatic Control, 48(11):2040-2045, 2003.

[17] J. P. Hespanha and A. S. Morse. Stability of switched systems with average dwell-time. In IEEE Conference on Decision and Control, pages 2655-2660, Phoenix, Arizona, 1999.

[18] K. Hirata and J. P. Hespanha. $\mathcal{L}_{2}$-induced gains of switched systems and classes of switching signals. In IEEE Conference on Decision and Control, pages 438-442, Atlanta, USA, 2010.

[19] H. Ishii, T. Basar, and R. Tempo. Randomized algorithms for synthesis of switching rules of multimodal systems. IEEE Transactions on Automatic Control, 50(6):754-767, 2005.

[20] M. Johansson and A. Rantzer. Computation of piecewise quadratic Lyapunov functions for hybrid systems. IEEE Transactions on Automatic Control, 43:555-559, 1998.

[21] D. Liberzon. Switching in Systems and Control. Birkhauser, Boston, 2003

[22] D. Liberzon and A. S. Morse. Basic problems in stability and design of switched systems. IEEE Control Systems Magazine, 19(5):59-70, 1999.

[23] M. Margaliot. Stability analysis of switched systems using variational principles: An introduction. Automatica, 42(12):2059-2077, 2006

[24] M. Margaliot and J. P. Hespanha. Root-mean-square gains of switched linear systems: a variational approach. Automatica, 44(9):2398-2402, 2008.

[25] A. S. Morse. Supervisory control of families of linear set-point controllers - part 1 : Exact matching. IEEE Transactions on Automatic Control, 41(10):1413-1431, 1996.

[26] J. F. Sturm. Using SeDuMi 1.02, a MATLAB toolbox for optimization over symmetric cones. Optimization Methods and Software, 11-12:625653, 1999.
[27] M. Wicks and R. A. DeCarlo. Solution of coupled Lyapunov equations for the stabilization of multimodal linear systems. In American Control Conference, pages 1709-1713, 1997.

[28] F. Wirth. A converse Lyapunov theorem for linear parameter varying and linear switching systems. SIAM Journal on Control and Optimization, 44:210-239, 2005

[29] K. Zhou and J. C. Doyle. Essential of Robust Control. Prentice Hall, 1997.

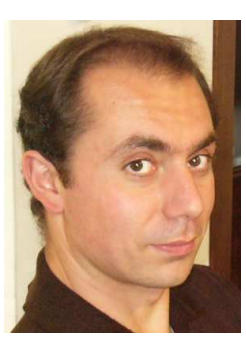

Graziano Chesi received the Laurea in Information Engineering from the University of Florence in 1997 and the PhD in Systems Engineering from the University of Bologna in 2001. He joined the University of Siena in 2000 and the University of Hong Kong in 2006. Dr. Chesi served as Associate Editor for Automatica, the European Journal of Control, the IEEE Control Systems Letters, the IEEE Transactions on Automatic Control, the IEEE Transactions on Computational Biology and Bioinformatics, and Systems and Control Letters. He also served as Guest Editor for the IEEE Transactions on Automatic Control, the International Journal of Robust and Nonlinear Control, and Mechatronics. Dr. Chesi founded and served as chair of the Technical Committee on Systems with Uncertainty of the IEEE Control Systems Society. He also served as chair of the Best Student Paper Award Committees for the IEEE Conference on Decision and Control and the IEEE Multi-Conference on Systems and Control. Dr. Chesi is author of the books "Homogeneous Polynomial Forms for Robustness Analysis of Uncertain Systems" (Springer 2009) and "Domain of Attraction: Analysis and Control via SOS Programming" (Springer 2011). He is a Fellow of the IEEE.

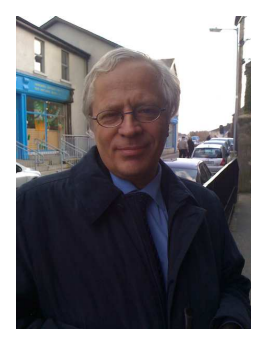

Patrizio Colaneri was born in Palmoli, Italy, in 1956. He received the Laurea degree in Electrical Engineering in 1981 and the Ph.D. degree (Dottorato di Ricerca) in Automatic Control in 1987. After a few years in industry and at the National Research Council of Italy, he joined the Politecnico di Milano where he is full professor of Automatica and served as head of the Ph.D. school on ICT (2007-2009). He spent a semester at the Systems Research Center of the University of Maryland (1989) and at the Hamilton Institute of the National University of Ireland (2009). He also collaborates with the Institute for Design and Control of Mechatronical Systems of Johannes Kepler University in Linz (Austria) since 2000. Dr. Colaneri was the chair of the IFAC Coordinating Committee on Design Methods for six years, the chair of the Technical Committee on Control Design for six years, a member of the Council of EUCA (European Union Control Association) for three years, an Associate Editor of Automatica for six years (certificate of outstanding service). He is at present a member of the Technical Board of IFAC, a Subject Editor of the International Journal of Robust and Nonlinear Control, a senior Editor of the IFAC journal Nonlinear Analysis Hybrid Systems and a Senior Editor of the IEEE Transactions on Automatic Control. He was elevated to the degree of IEEE Fellow for contributions on periodic and switching control. He is also a Fellow of IFAC (International Federation of Automatic Control). His main interests are in the area of periodic systems and control, robust filtering and control, and switching control. He has authored/co-authored more than 220 papers and seven books, including the following four international monographies: "Control Theory and Design: an RH2 and RH $\infty$ viewpoint", Academic Press 1997, "Periodic Systems: Filtering and Control", Springer Verlag, 2009, "Positive Markov Jump Linear Systems", Now Publishing 2015, "Switched Positive Linear Systems", Now Publishing 2015. 